\title{
Chemical modelling of dust-gas chemistry within AGB outflows III. Photoprocessing of the ice and return to the ISM
}

\author{
M. Van de Sande ${ }^{1 \star} \dagger$, C. Walsh ${ }^{2} \&$ T.J. Millar ${ }^{3}$ \\ ${ }^{1}$ Institute of Astronomy, KU Leuven, Celestijnenlaan 200D, 3001 Leuven, Belgium \\ ${ }^{2}$ School of Physics and Astronomy, University of Leeds, Leeds LS2 9JT, UK \\ ${ }^{3}$ Astrophysics Research Centre, School of Mathematics and Physics, Queen's University Belfast, University Road, Belfast BT7 1NN, UK
}

Accepted XXX. Received YYY; in original form ZZZ

\begin{abstract}
To explain the properties of dust in the interstellar medium (ISM), the presence of a refractory organic mantle is necessary. The outflows of AGB stars are among the main contributors of stellar dust to the ISM. We present the first study of the refractory organic contribution of AGB stars to the ISM. Based on laboratory experiments, we included a new reaction in our extended chemical kinetics model: the photoprocessing of volatile complex ices into inert refractory organic material. The refractory organic feedback of AGB outflows to the ISM is estimated using observationally motivated parent species and grids of models of C-rich and O-rich outflows. Refractory organic material is mainly inherited from the gas phase through accretion onto the dust and subsequent photoprocessing. Grain-surface chemistry, initiated by photodissociation of ices, produces only a minor part and takes place in a sub-monolayer regime in almost all outflows. The formation of refractory organic material increases with outflow density and depends on the initial gas-phase composition. While O-rich dust is negligibly covered by refractory organics, C-rich dust has an average coverage of $3-9 \%$, but can be as high as $8-22 \%$. Although C-rich dust does not enter the ISM bare, its average coverage is too low to influence its evolution in the ISM or significantly contribute to the coverage of interstellar dust. This study opens up questions on the coverage of other dust-producing environments. It highlights the need for an improved understanding of dust formation and for models specific to density structures within the outflow.
\end{abstract}

Key words: Stars: AGB and post-AGB - circumstellar matter - astrochemistry molecular processes - ISM: molecules - ISM: dust, extinction

\section{INTRODUCTION}

Interstellar dust is thought to have a layered structure of a silicate core, an inner organic mantle, and an outer ice mantle. The inner organic mantle consists of refractory organic material containing a mixture of aliphatic and aromatic carbonaceous molecules, formed by the photoprocessing of interstellar ices during their evolution in the interstellar medium (ISM) (Greenberg \& Shen 1999; Jones et al. 2013). This inner refractory organic grain mantle is necessary to explain the amount and wavelength dependence of extinction, for which silicate grains alone do not suffice. A combination of silicates with graphite can mediate this (Mathis 1979), but the polarisation of the extinction demands a coating of refractory organic material containing $\mathrm{O}$,

\footnotetext{
* E-mail: marie.vandesande@kuleuven.be

$\dagger$ Postdoctoral Fellow of the Fund for Scientific Research (FWO),

Flanders, Belgium
}

C and N. (Greenberg 1986). Observational evidence comes mainly from the aliphatic $\mathrm{CH}$ stretching-mode band at 3.4 $\mu \mathrm{m}$ (e.g., Willner et al. 1979; Butchart et al. 1986; Sandford et al. 1991; Duley et al. 1998; Pendleton \& Allamandola 2002). It has also been proposed as a potential carrier of the unidentified depleted oxygen in the ISM (Whittet 2010).

Laboratory experiments have studied the formation of refractory organic material by exposing interstellar ice analogues to UV radiation. When heating up the processed dust analogues, refractory organic material containing large molecules, carbonaceous material and even amino acids, stays behind (e.g., Greenberg et al. 1972; Hagen et al. 1979; Allamandola et al. 1988; Bernstein et al. 2002; Muñoz Caro \& Schutte 2003; Materese et al. 2017). The refractory organic mantle has been found to change the properties of the dust. For example, coated silicates have a much higher sticking threshold velocity (Kouchi et al. 2002), and the coating can increase the tensile strength of the dust grains while not inherently changing its stickiness (Bischoff et al. 2020). 
The outflows of Asymptotic Giant Branch (AGB) stars are important contributors to the chemical enrichment of the ISM. Together with supernovae, they are the main contributors of pristine stellar dust to the ISM (Zhukovska \& Henning 2013). The outflow is thought to be launched in a two-step mechanism, where stellar pulsations facilitate dust formation, which then launches a dust-driven wind (Höfner \& Olofsson 2018). Exactly how the dust is formed is still unknown. This implies that several characteristics of AGB dust are not known as it enters the ISM, such as its exact composition and its grain-size distribution. These characteristics are crucial to our understanding of the physical and chemical dust evolution cycle in the ISM.

The chemical kinetics model developed in Van de Sande et al. $(2019,2020)$ (henceforth Papers I and II, respectively) is the first to consider dust-gas interactions and grain-surface chemistry in AGB outflows. We found that gas-phase species can be efficiently depleted onto the dust, forming an ice mantle. However, the chemical reaction network included only a simple surface chemistry, which limits the possible complexity of ice species. The model also did not take the irradiation of the ice mantle into account and any photoprocessing that could occur in the outer regions of the outflow. Given that our previous models predict efficient depletion of gas-phase species onto the dust, this raises the possibility of AGB outflows hosting a feedstock of volatile organic molecules on grain surfaces that could be photoprocessed into refractory organic material, as found in laboratory experiments.

Here, we present the first study of the contribution of refractory organic material from AGB outflows to the ISM. To do so, a complex surface chemistry was included, along with photodissociation on the grain surface and reactive desorption. We introduce photoprocessing of complex volatile ice species into inert refractory organic material, a first in chemical modelling of AGB outflows, using the results of laboratory experiments. Based on observational samples of AGB stars, we assembled grids of C-rich and O-rich outflows. We also compiled new observationally motivated sets of parent species. This way, we are able to provide the first estimation of the refractory organic coverage of AGB dust as it enters the ISM, forming the starting point of its subsequent interstellar evolution. This gives a more comprehensive view on the starting point of dust evolution in the ISM.

The new chemical reaction network and the new reactions included in the model are described in Sect. 2, along with the compilation of observational grids of C-rich and O-rich outflows and of the observationally motivated sets of parent species. Our results on the formation of refractory organic material within AGB outflows and their contribution to the ISM are shown in Sect. 3. This is followed by their discussion in Sect. 4 and the conclusions in Sect. 5 .

\section{THE CHEMICAL MODEL}

The one-dimensional chemical kinetics model is fully described in Papers I and II. Details of the model can be found in Appendix A. We use the chemical reaction network of Walsh et al. (2014) designed for use in protoplanetary disk chemical models. This includes the full gas-phase network UDfA RATE12 (McElroy et al. 2013) supplemented with a set of grain-surface reactions and associated processes, in-
Table 1. Parameters of the grid of chemical models.

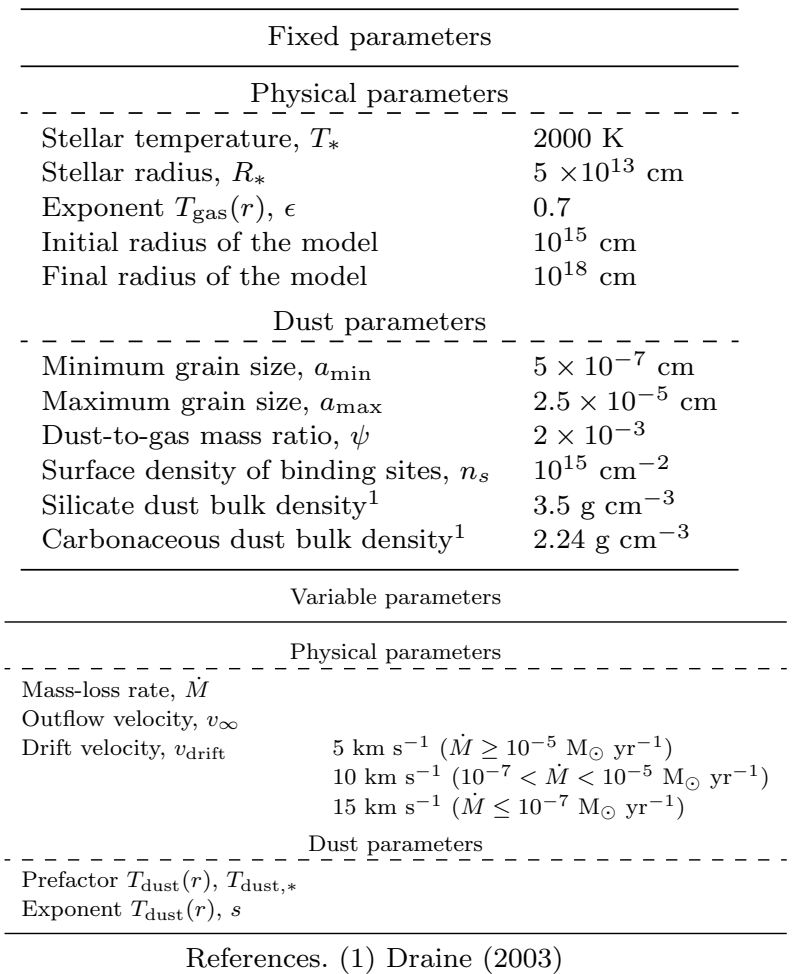

cluding needed gas-phase reactions, extracted from the 2008 version of Ohio State University (OSU) network (Garrod et al. 2008). The surface-chemistry network includes atomaddition reactions to form both simple (e.g., water, $\mathrm{H}_{2} \mathrm{O}$ ) and more complex ices (e.g., methanol, $\mathrm{CH}_{3} \mathrm{OH}$ ) as well as radical-radical recombination reactions that help to build larger molecules (e.g., glycolaldehyde, $\left.\left(\mathrm{CH}_{2} \mathrm{OH}\right)_{2}\right)$. The network also includes thermal and non-thermal desorption processes (photodesorption, reactive desorption, and sputtering) and includes the dissociation of ice species on the surface via photodissociation and photoionisation by both external photons and those generated internally by cosmic rays.

Table 1 lists all parameters describing the outflow and dust. The gas temperature profile does not change throughout the modelling. The gas-to-dust mass ratio and the surface density of binding sites are kept constant. The dustgrain size distribution (GSD) is fixed to the canonical MRN distribution (Mathis et al. 1977). The dust bulk density depends on whether the outflow is C-rich or O-rich. The outer radius of the model depends on the outflow density, and is taken to be where the $\mathrm{H}_{2}$ number density reaches $10 \mathrm{~cm}^{-3}$, i.e., where the outflow is assumed to merge with the ISM.

The new grain-surface reactions included in the model are described in Sect. 2.1. The observational set of AGB outflows is described in Sect. 2.2, together with the observational motivation behind the assumed drift velocities and dust compositions. The new observationally motivated sets of parent species are presented in Sect. 2.3.

\subsection{New grain-surface reactions}

Including a more complex chemistry requires adding grainsurface reactions to render the network more complete: reac- 


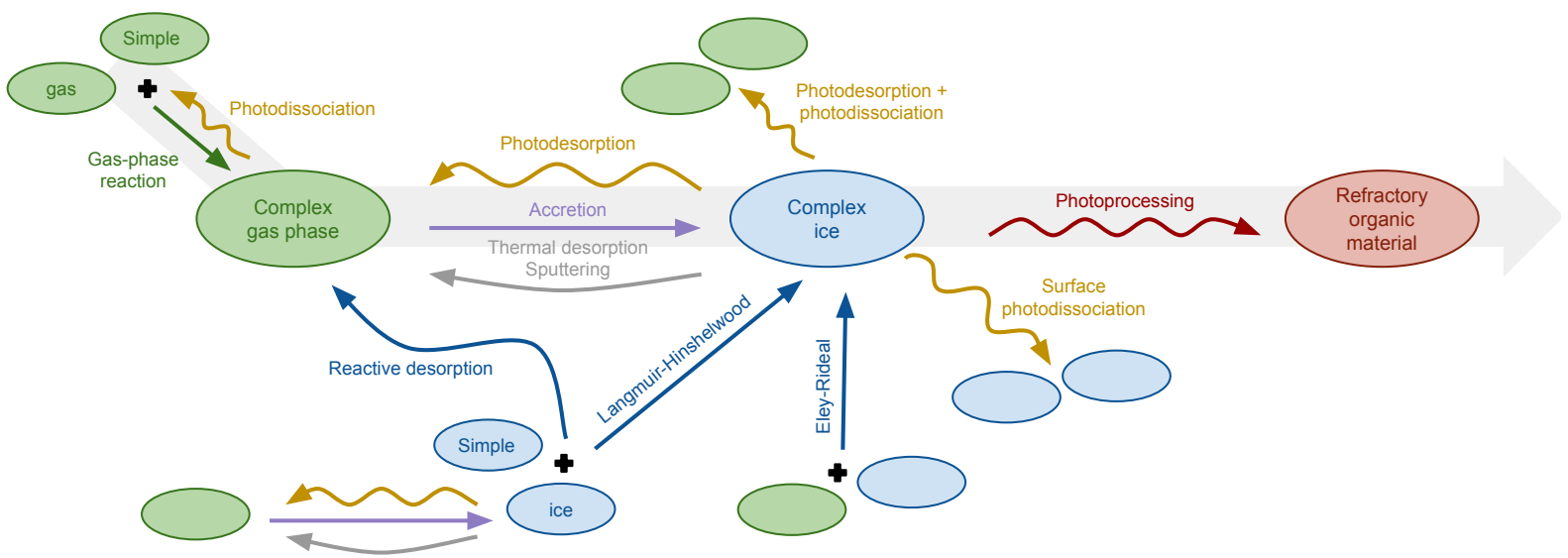

Figure 1. Scheme representing the different formation and destruction pathways included in the chemical network. Gas-phase reactions are simplified in this scheme. Photoprocessing of complex ice species into inert refractory organic material creates a sink of C-bearing material on the dust grain. Green ellipses: gas-phase species, blue ellipses: ice species, red ellipse: refractory organic material. The main pathway to the formation complex ices and refractory organic material is highlighted by the grey arrow.

tive desorption and photodissociation of ices on the surface. This is done following the recipes of Cuppen et al. (2017) for models using the mean-field rate-equation approach. The influence of these new reactions on the chemistry is discussed in Appendix B. We also include a new type of reaction, the photoprocessing of volatile ice species to inert refractory organic material. Fig. 1 illustrates all pathways included in the network, focussing on the grain-surface chemistry.

Reactive desorption Grain-surface reactions, via either the diffusive Langmuir-Hinshelwood or the stick-and-hit EleyRideal mechanism, can result in desorption of the product into the gas phase because of excess reaction energy. The reactive desorption rate is calculated as

$k_{\mathrm{RD}}=B k_{\mathrm{LH} / \mathrm{ER}} \mathrm{s}^{-1}$,

where $k_{\mathrm{LH} / \mathrm{ER}}$ is the Langmuir-Hinshelwood or the EleyRideal reation rate (Paper I) and $B$ is the reactive desorption efficiency. We assume that $B=0.01$ throughout our calculations.

Photodissociation on the grain surface Photodissociation on the grain surface is treated as a parallel process to photodesorption. The reaction rates of photodissociation caused by UV photons or cosmic-ray induces photoreactions are those given in McElroy et al. (2013).

Photoprocessing to refractory organic material The photoprocessing of volatile ice to inert refractory organic material is limited to complex species only, which are defined as species containing at least three $\mathrm{C}$ atoms or at least two $\mathrm{C}$ atoms and one other heavy atom $(\mathrm{N}, \mathrm{O}, \mathrm{Si}$, or $\mathrm{S})$. No back reaction is included for refractory organic material, so that photoprocessing creates a sink for complex ice species and removes any further possible reactions between them. Polymerisation is not explicitly included in the network, but is implied as part of the conversion from volatile to refractory organics. The refractory organics do not react among themselves, nor with ice and gas-phase species, and hence are being treated as chemically inert. The photoprocessing rate

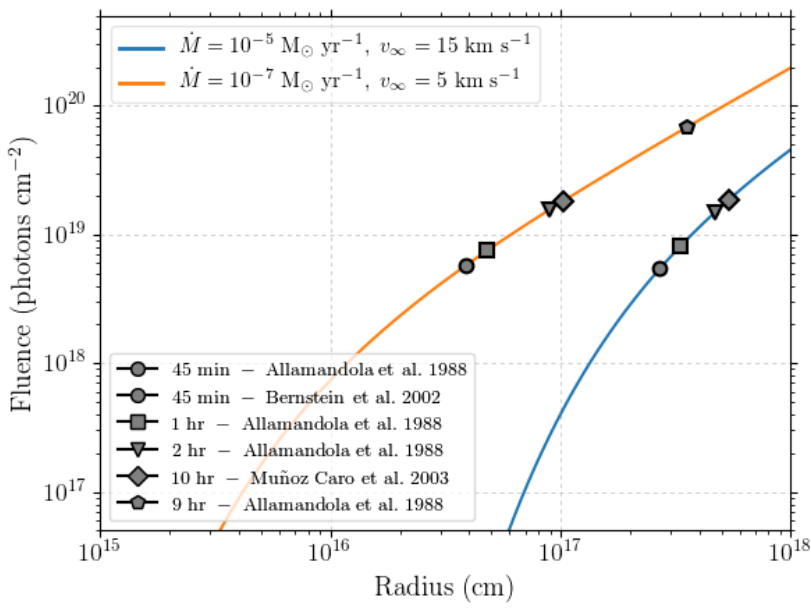

Figure 2. Fluence throughout a high density outflow $\left(\dot{M}=10^{-5}\right.$ $\mathrm{M}_{\odot} \mathrm{yr}^{-1}, v_{\infty}=15 \mathrm{~km} \mathrm{~s}^{-1}$, blue line) and a low density outflow $\left(\dot{M}=10^{-7} \mathrm{M}_{\odot} \mathrm{yr}^{-1}, v_{\infty}=5 \mathrm{~km} \mathrm{~s}^{-1}\right.$, orange line). Markers: fluences obtained in lab experiments after a certain exposure time.

is similar to that of photodissociation,

$k_{\mathrm{VR}}=\omega \exp \left(-\gamma A_{V}\right) \quad \mathrm{s}^{-1}$,

where $\omega$ is the photoprossessing rate to refractory organic material in the unshielded interstellar UV field, $A_{V}$ is the dust extinction at visible wavelengths, and $\gamma$ is a parameter that takes the increased dust extinction at UV wavelengths into account (McElroy et al. 2013). We have assumed that $\gamma$ is equal to that of $\mathrm{H}_{2} \mathrm{O}$, which is generally the largest ice component. This value is also similar to many C-bearing molecules in ices.

The value of $\omega$ is empirically estimated from laboratory experiments. Fig. 2 shows fluences used in experiments by Allamandola et al. (1988), Bernstein et al. (2002), and Muñoz Caro \& Schutte (2003) together with the fluence of interstellar UV photons in a low- and high-density outflow. This is calculated by integrating the flux of interstellar UV photons throughout the outflow over time, done by multi- 
Table 2. Parent species and their initial abundances relative to $\mathrm{H}_{2}$. Upper part: O- and C-rich parents of Papers I and II. Lower part: C-rich parent species as derived from observations (Agúndez et al. 2020). Left column: mean abundances (ab.) from the observed ranges. Right column: mean abundances, with the maximal observed abundance for C-bearing species.

\begin{tabular}{|c|c|c|c|}
\hline \multicolumn{4}{|c|}{ Parent species used in Papers I \& II } \\
\hline \multicolumn{2}{|c|}{ Oxygen-rich } & \multicolumn{2}{|c|}{ Carbon-rich } \\
\hline $\mathrm{He}$ & 0.17 & $\mathrm{He}$ & 0.17 \\
\hline $\mathrm{CO}$ & $3.0 \times 10^{-4}$ & $\mathrm{CO}$ & $8.0 \times 10^{-4}$ \\
\hline $\mathrm{N}_{2}$ & $4.0 \times 10^{-5}$ & $\mathrm{~N}_{2}$ & $4.0 \times 10^{-5}$ \\
\hline $\mathrm{H}_{2} \mathrm{O}$ & $3.0 \times 10^{-4}$ & $\mathrm{C}_{2} \mathrm{H}_{2}$ & $8.0 \times 10^{-5}$ \\
\hline $\mathrm{CO}_{2}$ & $3.0 \times 10^{-7}$ & $\mathrm{HCN}$ & $2.0 \times 10^{-5}$ \\
\hline $\mathrm{SiO}$ & $5.0 \times 10^{-5}$ & $\mathrm{SiO}$ & $1.2 \times 10^{-7}$ \\
\hline $\mathrm{SiS}$ & $2.7 \times 10^{-7}$ & $\mathrm{SiS}$ & $1.0 \times 10^{-6}$ \\
\hline $\mathrm{SO}$ & $1.0 \times 10^{-6}$ & CS & $5.0 \times 10^{-7}$ \\
\hline $\mathrm{H}_{2} \mathrm{~S}$ & $7.0 \times 10^{-8}$ & $\mathrm{SiC}_{2}$ & $5.0 \times 10^{-8}$ \\
\hline $\mathrm{PO}$ & $9.0 \times 10^{-8}$ & $\mathrm{HCP}$ & $2.5 \times 10^{-8}$ \\
\hline $\mathrm{HCN}$ & $2.0 \times 10^{-7}$ & $\mathrm{NH}_{3}$ & $2.0 \times 10^{-6}$ \\
\hline $\mathrm{NH}_{3}$ & $1.0 \times 10^{-7}$ & $\mathrm{H}_{2} \mathrm{O}$ & $1.0 \times 10^{-7}$ \\
\hline
\end{tabular}

Parent species compiled from Agúndez et al. (2020)

Carbon-rich, mean observed ab.

\begin{tabular}{ll}
\hline $\mathrm{He}$ & $1.70 \times 10^{-1}$ \\
$\mathrm{CO}$ & $8.00 \times 10^{-4}$ \\
$\mathrm{~N}_{2}$ & $4.00 \times 10^{-5}$ \\
$\mathrm{C}_{2} \mathrm{H}_{2}$ & $4.38 \times 10^{-5}$ \\
$\mathrm{HCN}$ & $4.09 \times 10^{-5}$ \\
$\mathrm{SiO}$ & $5.02 \times 10^{-6}$ \\
$\mathrm{SiS}$ & $5.98 \times 10^{-6}$ \\
$\mathrm{CS}$ & $1.06 \times 10^{-5}$ \\
$\mathrm{SiC}_{2}$ & $1.87 \times 10^{-5}$ \\
$\mathrm{HCP}$ & $2.50 \times 10^{-8}$ \\
$\mathrm{NH}_{3}$ & $6.00 \times 10^{-8}$ \\
$\mathrm{H}_{2} \mathrm{O}$ & $2.55 \times 10^{-6}$ \\
$\mathrm{CH}_{4}$ & $3.50 \times 10^{-6}$ \\
$\mathrm{HCl}^{-6}$ & $3.25 \times 10^{-7}$ \\
$\mathrm{C}_{2} \mathrm{H}_{4}$ & $6.85 \times 10^{-8}$ \\
$\mathrm{HF}$ & $1.70 \times 10^{-8}$ \\
$\mathrm{H}_{2} \mathrm{~S}$ & $4.00 \times 10^{-9}$ \\
\hline
\end{tabular}

plying the flux at a specific radius within the outflow by the expansion time, which is the radius divided by the outflow velocity. The fluences needed to convert volatile ices to inert refractory organic material are reached in the outer region, at $\sim 10^{17} \mathrm{~cm}$, for both outflow densities. The fluence corresponding to a $37 \mathrm{~h}$ exposure (Allamandola et al. 1988) is achieved outside the outflow and not visible in Fig. 2. From the exposure times, we find that the photoprocessing rate $\omega$ lies between $3 \times 10^{-4}-8 \times 10^{-6} \mathrm{~s}^{-1}$. We have taken $\omega=10^{-5}$ $\mathrm{s}^{-1}$ as a reasonable value for the photoprocessing rate.

\subsection{Observational grids of AGB outflows}

In Papers I and II, we assumed representative outflow densities for the model calculations. In this work, we used observational studies of AGB outflows, where mass-loss rate and outflow velocity were retrieved, to compile grids of O-rich and C-rich outflows. These observationally motivated grids

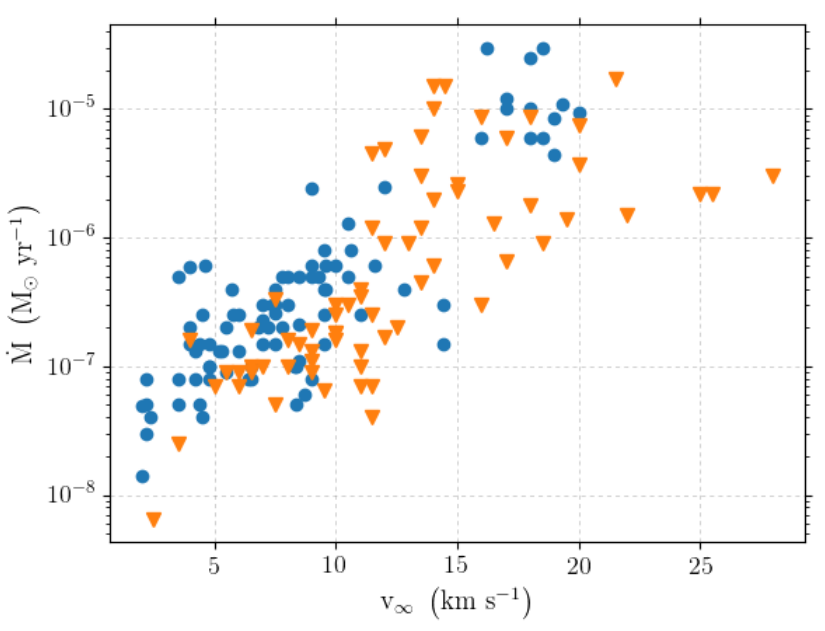

Figure 3. Measured mass-loss rate, $\dot{M}$, and outflow velocity, $v_{\infty}$, of the AGB outflows included in the observational grids. Blue circles: O-rich outflows (Olofsson et al. 2002; González Delgado et al. 2003; Danilovich et al. 2015). Orange triangles: C-rich outflows (Schöier \& Olofsson 2001; Danilovich et al. 2015).

are necessary to estimate the refractory organic output of AGB stars to the ISM.

The sample of O-rich outflows is taken from Olofsson et al. (2002), González Delgado et al. (2003), and Danilovich et al. (2015). For C-rich outflows, we used Schöier \& Olofsson (2001) and Danilovich et al. (2015). If a star was present in multiple datasets, we have taken the measured mass-loss rate and outflow velocity from the most recent one. Fig. 3 shows the compiled O-rich and C-rich observational data sets.

The drift velocity between dust and gas is not readily retrieved from observations. Therefore, only a few outflows have a measured drift velocity (e.g., Groenewegen 1997; Decin et al. 2010; Van de Sande et al. 2018). In general, higher mass-loss rates are expected to have lower drift velocities. Together with the sample of Ramstedt et al. (2008), we have chosen for $v_{\text {drift }}=5 \mathrm{~km} \mathrm{~s}^{-1}$ if $\dot{M} \geq 10^{-5} \mathrm{M}_{\odot} \mathrm{yr}^{-1}$, $v_{\text {drift }}=10 \mathrm{~km} \mathrm{~s}^{-1}$ if $10^{-7}<\dot{M}<10^{-5} \mathrm{M}_{\odot} \mathrm{yr}^{-1}$, and $v_{\text {drift }}=15 \mathrm{~km} \mathrm{~s}^{-1}$ if $\dot{M} \leq 10^{-7} \mathrm{M}_{\odot} \mathrm{yr}^{-1}$.

The assumed dust composition is also based on observations. Heras \& Hony (2005) derived the composition of the dust around 28 O-rich AGB stars and found that Fe-bearing silicates are present in all outflows. Its total fraction depends on the outflow, as does the general composition of the dust. For the O-rich models, we assume that the dust is composed of a 50/50 mixture of melilite and silicate with Fe. This simplification roughly accounts for some of the variation seen in O-rich dust. However, it is necessary to calculate models for the observational sets, as the dust composition is not known for each outflow. For the C-rich models, we assume amorphous carbon as the only dust species. While SiC dust has been observed around C-rich AGB stars (e.g., Yang et al. 2004), we can assume that its temperature is similar to that of amorphous carbon as their overall wavelength-dependence of absorption is similar (Rau et al. 2017). The calculation of the dust temperature profiles is detailed in Appendix C.

The measured mass-loss rates and outflow velocities, assumed drift velocities, and parameters of the dust tempera- 


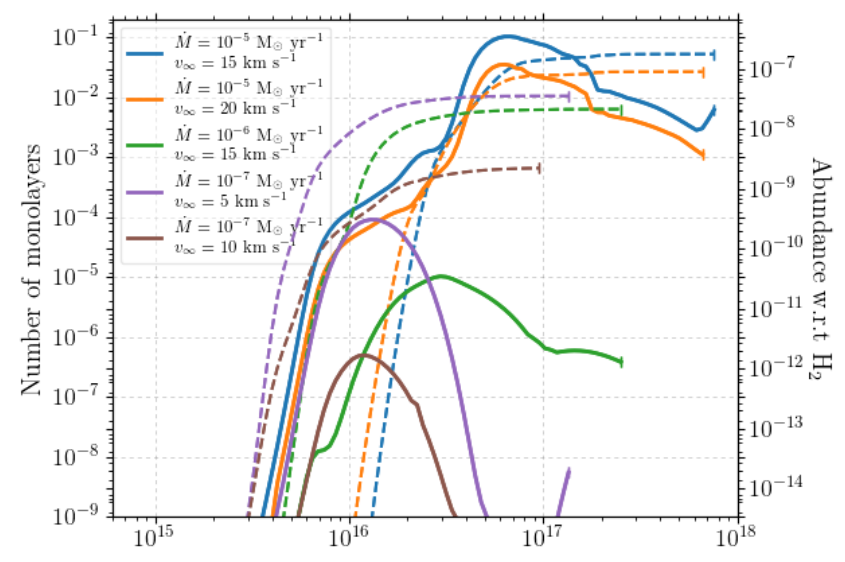

Figure 4. Number of monolayers (left y-axis) and abundance relative to $\mathrm{H}_{2}$ (right $\mathrm{y}$-axis) of volatile ice (solid lines) and refractory organic material (dashed lines) in C-rich outflows with different mass-loss rates and outflow velocities, using the parent species of Papers I and II. The dash marks where the $\mathrm{H}_{2}$ number density reaches $10 \mathrm{~cm}^{-3}$, i.e., where the outflow merges with the ISM.

ture profiles are listed in Tables C.1 and C.2 for the grid of C-rich and O-rich outflows, respectively.

\subsection{Parent species}

Table 2 lists the sets of parent species used. In addition to the O- and C-rich parent species of Papers I and II, we compiled two observationally motivated sets for C-rich outflows. These are based on Agúndez et al. (2020), who have compiled (ranges of) observed abundances of parent molecules in C-rich, O-rich, and S-type stars (see their Table 2). One set uses the mean of the ranges in observed abundances, as an approximation of an average C-rich outflow. The other has the maximal abundances for C-bearing species to maximise the possible refractory organic feedstock, to explore an extreme case.

\section{RESULTS}

Refractory organic material can be formed on the dust within AGB outflows. The majority of refractory organic material is inherited from the gas phase: complex species are formed in the gas phase, accreted onto the dust and then photoprocessed into refractory organic material over the timescale of the outflow (highlighted in Fig. 1 by a grey arrow). Complex ice species can also be formed through grain-surface chemistry. They too can be subsequently photoprocessed, but make up only a minority of the refractory organic material injected into the ISM.

The outflow density and the initial gas-phase composition play a crucial role: (i) The outflow density determines the accretion rate and the onset of photon-reactions (photodissociation in the gas phase and the ice, photodesorption and photoprocessing). The onset shifts closer to the star as the outflow density decreases. Before the onset of photon-reactions, the ice mantle is directly inherited from
Table 3. Outflow-density weighted average refractory organic output of C-rich outflows for different parent species, together with the average number of refractory organic molecules per grain and their fractional abundance with respect to $\mathrm{H}_{2}$ in the ISM.

\begin{tabular}{llll}
\hline & Papers I \& II & Mean obs. & $\begin{array}{l}\text { Mean obs. } \\
\text { max C }\end{array}$ \\
\hline Mean N mono & $5.66 \times 10^{-2}$ & $3.56 \times 10^{-2}$ & $9.45 \times 10^{-2}$ \\
Mean \# per grain & $7.64 \times 10^{2}$ & $4.80 \times 10^{2}$ & $1.27 \times 10^{3}$ \\
Frac. ab. in ISM & $7.64 \times 10^{-10}$ & $4.80 \times 10^{-10}$ & $1.27 \times 10^{-9}$ \\
\hline
\end{tabular}

the gas phase through accretion. After the onset, the accreted complex ice species can be photoprocessed into refractory organic material. They can also be photodissociated into simpler and radical ices, starting the minor grainsurface chemistry formation route to complex ices and subsequently refractory organic material. (ii) The initial gasphase composition determines the availability of gas-phase and icy feedstock material. C-rich outflows inherently provide more feedstock. The initial abundances of $\mathrm{HCN}$ and $\mathrm{C}_{2} \mathrm{H}_{2}$, especially, are essential. Conversely, O-rich outflows show a much smaller refractory organic coverage, linked to dearth of C-bearing parent species.

The refractory organic output to the ISM is limited. O-rich dust is barely covered, while $3-9 \%$ of the surface of C-rich dust is on average covered by refractory organic material. Nonetheless, photoprocessing of volatile ice to inert refractory organic material is essential to the long-term survival of the gas-phase species accreted and processed throughout the outflow. Without it, the entire ice mantle would be ablated as it enters the ISM.

In Sects. 3.1 and 3.2, we present the results for C-rich and O-rich outflows, respectively. First, the formation of refractory organic material in representative outflows is shown, followed by a quantification of the refractory organic output of the observational grids to the ISM.

\subsection{C-rich outflows}

Fig. 4 shows the number of monolayers of volatile ice and inert refractory organic material in different C-rich outflows together with their total abundance relative to $\mathrm{H}_{2}$, using the parent species of Papers I and II. The outflow densities are based on the observational grid and are representative for C-rich AGB stars. The influence of the outflow density can be clearly seen. The highest density outflow has a peak number of ice monolayers of $\sim 0.1$, more than five orders of magnitude larger than in the lowest density outflow. Surface chemistry hence takes place in a sub-monolayer regime. Consequently, the highest density outflow has $\sim 0.05$ monolayers of refractory organic material, which corresponds to a coverage of $\sim 5 \%$, while the lowest density outflow has a refractory organic coverage of less than $0.1 \%$. The onset of photoprocessing shifts closer to the star with decreasing outflow density. Feedstock ices are therefore photoprocessed more efficiently in lower density outflows, causing their rapid increase in refractory organic material. Therefore, the number of refractory organic monolayers differs only by about two orders of magnitude between the highest and the lowest density outflows, compared to more than five orders of magnitude for the number of ice monolayers. 

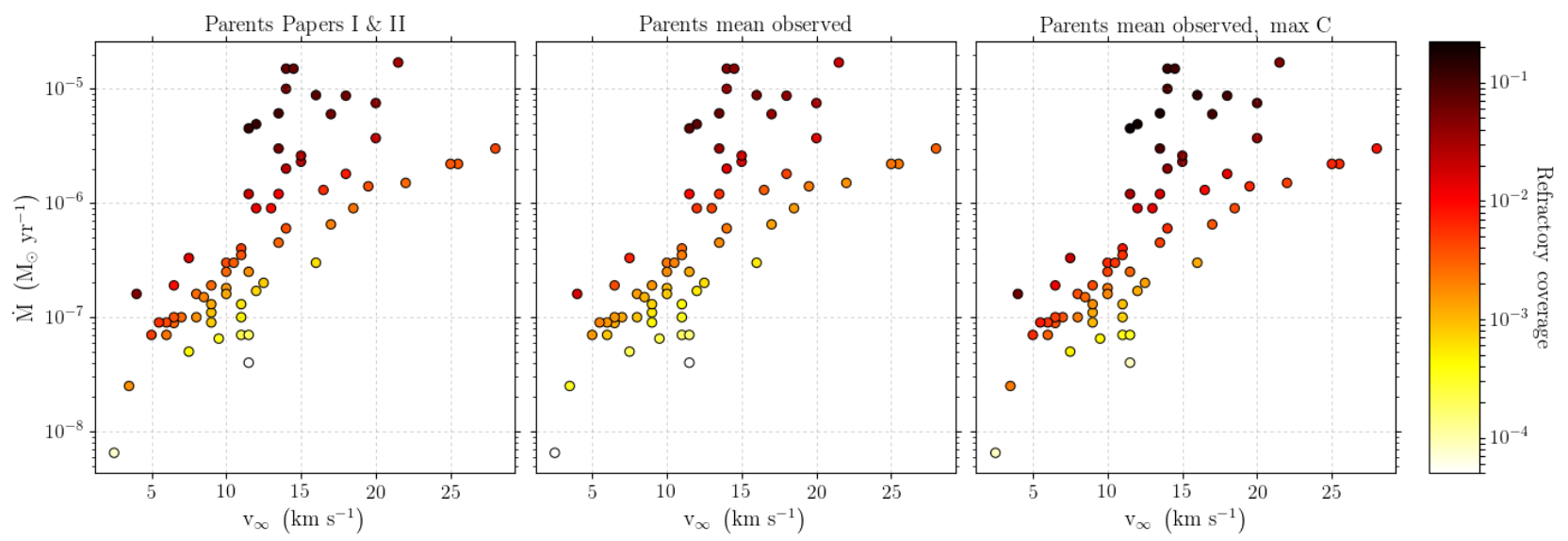

Figure 5. Refractory organic coverage of the dust grains in the outflows of the C-rich observational grid, for different sets of parent species (Table 2). The coverage corresponds to the number of refractory organic monolayers where the outflow merges with the ISM, which is taken to be the radius where $n\left(\mathrm{H}_{2}\right) \leq 10 \mathrm{~cm}^{-3}$. Left panel: parent species used in Papers I and II. Middle panel: mean observed abundances from Agúndez et al. (2020). Right panel: mean observed abundances, with the maximal observed value for C-bearing species.
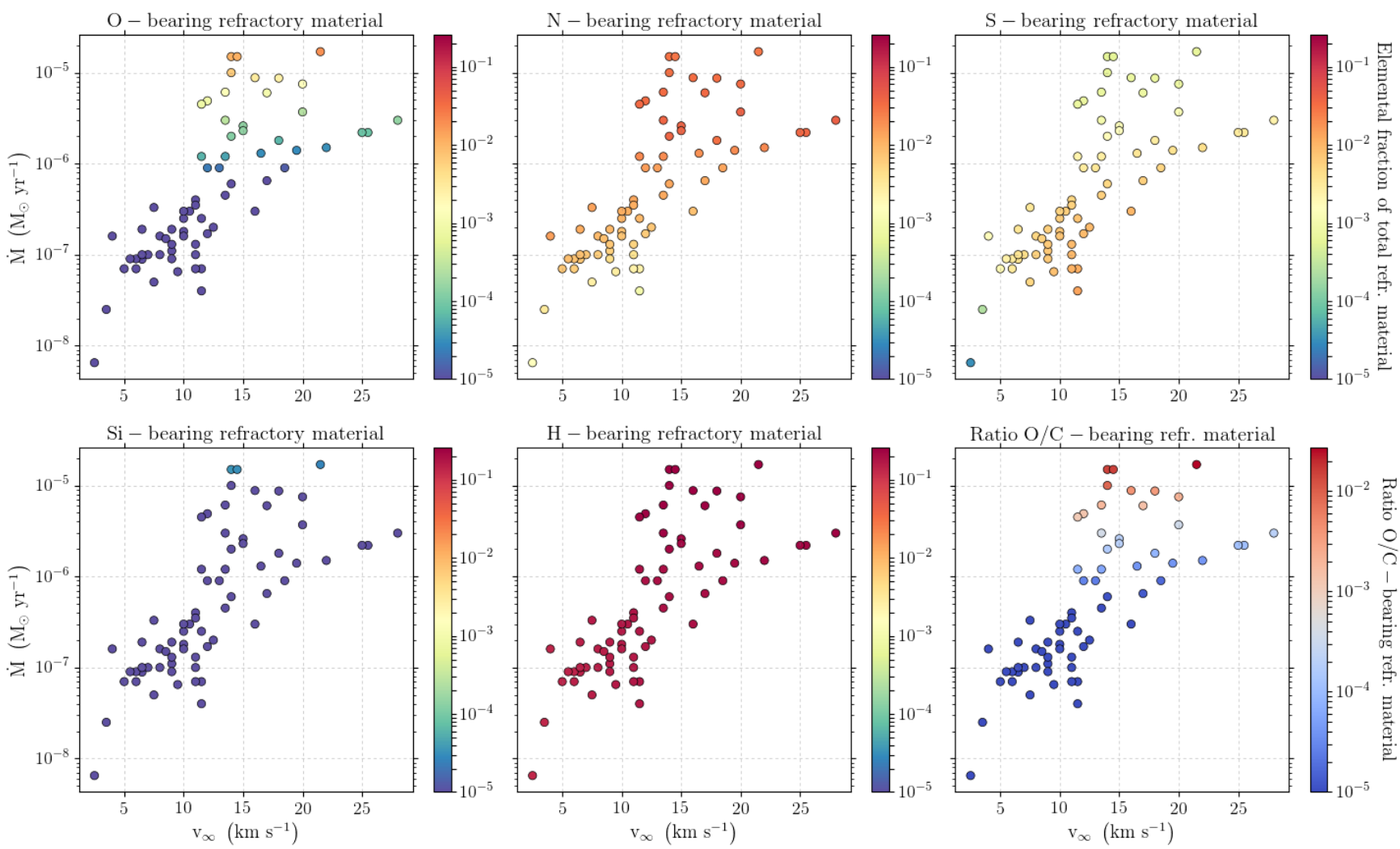

Figure 6. First five panels: elemental fractional composition of the total refractory organic output (O-, N-, S-, Si-, and H-bearing refractory organic material) for the outflows of the C-rich observational dataset. The parent species used are the mean observed abundances from Agúndez et al. (2020). Lower right panel: ratio of O-bearing/C-bearing refractory organic material of the total refractory organic output.

Fig. 5 shows the refractory organic coverage of the dust in the C-rich observational grid, using the three sets of parent species (Table 2). The coverage corresponds to the percentage of the surface covered by refractory organic material where the outflow merges with the ISM, which is taken to be the radius where $n\left(\mathrm{H}_{2}\right) \leq 10 \mathrm{~cm}^{-3}$. The coverage increases with increasing outflow density, achieved via a larger mass- loss rate or a lower outflow velocity. The maximum refractory organic coverage ranges from $8-22 \%$, where the range is due to the different sets of parent species. We find that $25-35 \%$ of the outflows in the C-rich grid have a refractory organic coverage larger than $1 \%$. A coverage larger than $10 \%$ is only achieved in up to $14 \%$ of the outflows. For CW Leo 


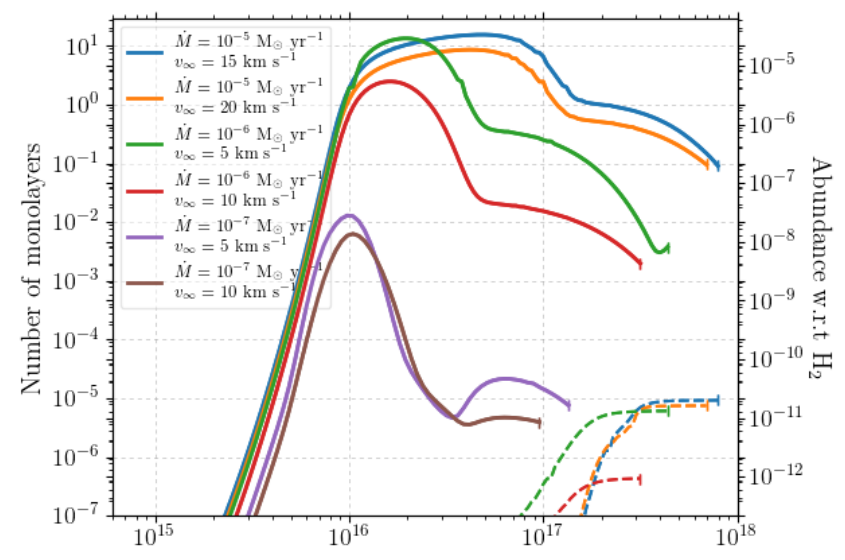

Figure 7. Number of monolayers (left y-axis) and abundance relative to $\mathrm{H}_{2}$ (right $y$-axis) of volatile ice (solid lines) and refractory organic material (dashed lines) in O-rich outflows with different mass-loss rates and outflow velocities. The dash marks where the $\mathrm{H}_{2}$ number density reaches $10 \mathrm{~cm}^{-3}$, i.e., where the outflow merges with the ISM.

(IRC+10216), the most studied C-rich AGB star, we find a refractory organic coverage between $5-11 \%$.

The dependency on the parent species is due to the relative abundances of $\mathrm{C}_{2} \mathrm{H}_{2}$ and $\mathrm{HCN}$. These are the main contributors of carbon and are crucial to the origin the gasphase complexity (e.g., Millar \& Herbst 1994; Agúndez et al. 2017). $\mathrm{C}_{2} \mathrm{H}_{2}$ is the key driver of chemical complexity, while $\mathrm{HCN}$ is necessary for the formation of $\mathrm{N}$-bearing $\mathrm{C}$-chains, e.g., cyanopolyynes. The mean observed parents with maximal carbon yield the largest refractory organic coverage as they have the largest $\mathrm{C}_{2} \mathrm{H}_{2}$ and $\mathrm{HCN}$ abundances. The parents of Papers I and II have the same $\mathrm{C}_{2} \mathrm{H}_{2}$ abundance but a lower HCN abundance, resulting in a smaller refractory organic coverage. The mean observed parents have the lowest $\mathrm{C}_{2} \mathrm{H}_{2}$ abundance and therefore the smallest refractory organic coverage, even though the HCN abundance is larger than that in Papers I and II.

Table 3 summarises the results of Fig. 5 and lists the outflow-density weighted average refractory organic output of the C-rich grid to the ISM. The average is calculated by weighing the mean by the mass-loss rate divided by the outflow velocity. The average number of refractory organic species per dust grain is calculated by multiplying the outflow-density weighted average number of monolayers by the average number of sites per grain. In the MRN GSD, the average cross-section of a grain is $\left\langle\sigma_{g}\right\rangle=3.37 \times 10^{-12}$ $\mathrm{cm}^{2}$, so that the average number of sites per grain is equal to $\Sigma_{\text {sites }}=4\left\langle\sigma_{g}\right\rangle n_{s}=1.35 \times 10^{4}$. Using the typical average dust density in the ISM of $10^{-12}$ relative to $\mathrm{H}_{2}$ (e.g., Tielens 2005 ), this can be converted to a fractional abundance of refractory organic material originating from AGB outflows in the ISM. The average outputs of the different sets of parent species lie within a factor two of each other.

Fig. 6 shows the elemental fractional composition of the refractory organic material in the C-rich outflows and its $\mathrm{O} / \mathrm{C}$ ratio, using the mean observed set of parent species. The elemental fractional composition is calculated per atom: for example, for $\mathrm{H}$, it is the total number of $\mathrm{H}$ atoms over
Table 4. Outflow-density weighted average refractory organic output of O-rich outflows, together with the average number of refractory organic molecules per grain and their fractional abundance with respect to $\mathrm{H}_{2}$ in the ISM.

\begin{tabular}{ll}
\hline & Papers I \& II \\
\hline Mean N mono & $6.28 \times 10^{-6}$ \\
Mean \# per grain & $8.47 \times 10^{-2}$ \\
Abundance in ISM & $8.47 \times 10^{-14}$ \\
\hline
\end{tabular}

all species divided by the total number of all atoms over all species. The amount of O-, N-, Si-, and H-bearing material increases with outflow density, while the amount of S-bearing material decreases. The refractory organic layer is poor in $\mathrm{Si}$, with generally less than $0.01 \%$ of the material locked up in $\mathrm{Si}$. An O-bearing component of $\sim 2 \%$ is only reached for the highest density outflows, with most outflows having a coverage lower than $\sim 0.01 \%$. The N-bearing component ranges from $\sim 5 \%$ to $\sim 0.1 \%$. The material is rich in $\mathrm{H}$, with between $10-25 \%$ of refractory organic material locked up in $\mathrm{H}$. The S-bearing fraction ranges from $\sim 1 \%$ for the lower density outflows to $\sim 0.1 \%$ for the higher density outflows. The $\mathrm{O} / \mathrm{C}$ ratio of the refractory organic material is generally low, reflecting the small fraction of O-bearing material. The ratio reaches values of approximately $10^{-2}$ only for the highest density outflows.

\subsection{O-rich outflows}

Fig. 7 shows the number of monolayers of volatile ice and inert refractory organic material, together with their total abundance relative to $\mathrm{H}_{2}$, for different O-rich outflows representative of the observational grid of O-rich AGB stars. The peak number of ice monolayers is larger than for the C-rich outflow, ranging from 10 monolayers for the highest density outflows to $\sim 10^{-2}$ monolayers for the lowest. This is due to the large initial $\mathrm{H}_{2} \mathrm{O}$ abundance, which has a large binding energy of $4880 \mathrm{~K}$ (see Paper I). The decrease in the ice mantle at large radii is caused by photodissociation on the grain surface. It does not correspond to an increase in refractory organic material, as in C-rich outflows (Fig. 4), because of the low carbon-content of the ice mantle. Although the ices used in laboratory experiments on refractory organic formation are also $\mathrm{H}_{2} \mathrm{O}$-rich, their carbon content is typically greater than $1 \%$. Only higher density outflows reach a refractory organic coverage of around $0.001 \%$. Both in the parent species of Papers I and II and those listed in Agúndez et al. (2020), $\mathrm{C}_{2} \mathrm{H}_{2}$ is not present and the $\mathrm{HCN}$ abundance is at least two orders of magnitude smaller than in C-rich outflows. Therefore only few C-bearing ices are present.

Table 4 lists the outflow-density weighted average refractory organic output of O-rich outflows to the ISM, summarising the results of the observational grid. The average coverage is four orders of magnitude smaller than that of Crich outflows. With on average 8.5 refractory organic species per dust grain, the coverage is negligible in the rate equation approach. The largest coverage is only $0.0015 \%$. The refractory organic coverage of the observational sample and its fractional composition are shown in Appendix E. 


\section{DISCUSSION}

Including photoprocessing of complex ice species in the chemical model leads to the build-up of refractory organic material. In AGB outflows, chemical complexity on the dust originates mainly from the gas phase: complex gas-phase species are accreted onto the dust and are subsequently photoprocessed. Active grain-surface chemistry plays only a minor role in the formation of complex ice species and refractory organic material. Complexity is not the result of simple ices reacting on the grain surface. This is unlike other environments where gas-grain chemistry takes place, such as dark clouds and protoplanetary disks (e.g., Henning \& Semenov 2013; Tielens 2013).

The grain-surface chemistry (photodissociation of ices and surface reactions between them) occurs in a submonolayer regime for the majority of the outflows. Submonolayer regimes are not common in astrochemical environments, holding only in the transition region from diffuse to dense interstellar clouds, where ice mantles are formed, and during the desorption of ice mantles at snowlines in protoplanetary disks (Potapov et al. 2019).

In Sect. 4.1, we discuss the origin of chemical complexity on the dust, together with the fractional composition of the refractory organic material. Sect. 4.2 focusses on the refractory organic contribution of AGB stars to the ISM and its impact on other astrochemical environments. Sect. 4.3 discusses the possible influence of surface effects on the refractory organic output. The sensitivity of our output to assumptions made in the model are discussed in Sect. 4.4. The influence of the new chemical network on the gas-phase chemistry is discussed in Appendix D.

\subsection{Refractory organic composition in C-rich outflows}

The effects of the two formation processes, accretion and surface chemistry, are visible in Fig. 6, which shows the fractional composition of the refractory organic material in the C-rich observational grid, using the mean observed set of parent species. The same processes are also at play in the O-rich outflows. As these outflows have significantly lower refractory organic coverage, they are discussed in Appendix E. In our notation, the prefix ' $G$ ' (grain) denotes ice species.

The S- and Si-bearing refractory organic species originate from accretion of gas-phase species and their subsequent photoprocessing only. This could be caused by the limited grain surface chemistry involving $\mathrm{S}$ and $\mathrm{Si}$ in the network. The S-bearing ices are limited to $\mathrm{GC}_{n} \mathrm{~S}(n=2,3,4)$, the Si-bearing ices to $\mathrm{GSiC}_{n}(n=2,3,4)$ and their hydrides. The fractional contribution of S-bearing species increases with decreasing outflow density, as this shifts the onset of photodissociation closer to the star, increasing the formation of gas-phase daughter species of the parent CS. The Si-bearing component increases with outflow density, but remains low $(\lesssim 0.1 \%)$. This is mainly due to the low binding energy of the parent $\mathrm{SiC}_{2}(1300 \mathrm{~K})$. The increase with outflow density is now caused by the shift of the onset photodissociation further away from the star, where $\mathrm{SiC}_{2}$ is less likely to thermally desorb upon accretion.

All O-bearing refractory organic species are solely formed in grain surface reactions, initiated by the photodissociation of ice species on the grain surface. Photodissoci- ation of $\mathrm{GH}_{2} \mathrm{O}$ results in $\mathrm{GO}$ and $\mathrm{GOH}$, two radicals that readily react. The main O-bearing component is formed via reactions with other simple radicals, such as $\mathrm{GC}_{n}(n=$ $1,2,3)$. More complex species are formed via a pathway of reactions involving complex ices and radicals, leading to refractory organic material composed of two $\mathrm{C}$ atoms and up to two $\mathrm{O}$ atoms. The fraction of O-bearing refractory organic material increases with outflow density because of the larger $\mathrm{GH}_{2} \mathrm{O}$ abundance, caused by the larger outflow density as well as the delay in photodissociation of $\mathrm{H}_{2} \mathrm{O}$. The increase in the O-bearing fraction leads to the increase in the $\mathrm{O} / \mathrm{C}$ ratio of the refractory organic material.

The N-bearing refractory organic species are formed by accretion and subsequent photoprocessing as well as grain surface reactions. Initially, $\mathrm{GHC}_{n} \mathrm{~N}(n=3,5,7,9)$ are formed through accretion. After the onset of photonreactions, they can be photoprocessed or photodissociated into $\mathrm{GC}_{n} \mathrm{~N}$, which can either reform $\mathrm{GHC}_{n} \mathrm{~N}$ or react with accreted $\mathrm{GCH}_{3}$ to form $\mathrm{GCH}_{3} \mathrm{C}_{n} \mathrm{~N}(n=3,5,7)$. These are the main N-bearing refractory organic species. Others are formed via accretion, with possible hydration on the surface, followed by photoprocessing. The fraction of N-bearing species increases with outflow density. This is linked to the gas-phase formation of $\mathrm{HC}_{n} \mathrm{~N}$. Because of their high binding energies $(4580 \mathrm{~K}, 6180 \mathrm{~K}, 7780 \mathrm{~K}, 9380 \mathrm{~K}$ for $n=3,5,7,9)$, they are readily depleted onto the dust close to the star for all outflow densities. Their peak gas-phase abundance shifts outwards with outflow density. This leads to a much broader peak in $\mathrm{GHC}_{n} \mathrm{~N}$ for higher density outflows, as the gas-phase $\mathrm{HC}_{n} \mathrm{~N}$ are already photodissociated in the lower density outflows, and therefore to a richer N-bearing grainsurface chemistry.

The fraction of H-bearing refractory organic species increases with outflow density. This is partly due to the increase with outflow density in N-bearing refractory organic species and the decrease with outflow density in S-bearing refractory organic species, which do not bear any $\mathrm{H}$. The most important factor is the influence of the onset of photonprocesses on the abundance of C-chains, with zero, one or two $\mathrm{H}$ atoms. In lower density outflows, the abundances of complex gas-phase daughter species increase closer to the star. This leads to a larger abundance of their ices, which are readily photoprocessed into refractory organic material, removing the possibility of hydrogenation on the grain surface and leading to a smaller $\mathrm{H}$-bearing component.

\subsection{Refractory organic contribution of AGB stars to the ISM and impact on other environments}

AGB stars are among the main contributors of dust and gas to the ISM, along with supernovae. C-rich AGB stars are estimated to inject dust into the Milky Way at a rate of $3 \mathrm{M}_{\odot} \mathrm{pc}^{-2} \mathrm{Myr}^{-1}$, which corresponds to $53 \%$ of the total C-rich dust injected. Similarly, O-rich AGB stars have an estimated silicate dust injection rate of $5 \mathrm{M}_{\odot} \mathrm{pc}^{-2} \mathrm{Myr}^{-1}$, corresponding to $29 \%$ of the total silicate dust injected (Tielens 2005). While we find that dust from O-rich outflows is covered by a negligible amount of refractory organic material, C-rich dust is on average covered by $3-9 \%$ of such material, depending on the parent species used. This implies that $53 \%$ of the C-rich dust in the Milky Way does not enter the ISM completely bare, but has some refractory 
organic material on its surface. However, the small average refractory organic coverage is unlikely to affect the physics and chemistry of the pristine dust entering the ISM. Using the parent species of Papers I and II and the observed parents with maximal C-bearing species, $5 \%$ and $14 \%$ of the outflows, respectively, have a refractory organic coverage of at least $10 \%$. The maximal coverage is $8 \%$ when using the mean observed parent species. Only in these high density outflows could the refractory organic coverage be able to significantly impact the dust's behaviour in the ISM surrounding the AGB star. However, further research is needed to fully quantify its possible impact.

Based on observational searches for refractory organic material, refractory organic material in the diffuse ISM is mainly composed of aromatic and aliphatic hydrocarbons with a minimal oxygen content, corresponding to a $\mathrm{O} / \mathrm{C}$ ratio $~ 0.015$ (Pendleton \& Allamandola 2002). The O-bearing component of the refractory organic material on C-rich dust is very low (Fig. 6). On average, over all outflows and all three sets of parent species, $\mathrm{O}$ only makes up $6.12 \times 10^{-4}$ of the refractory organic material, corresponding to a $\mathrm{O} / \mathrm{C}$ ratio of $\sim 9 \times 10^{-4}$, i.e., very depleted in oxygen. Therefore, the refractory organic contribution of C-rich AGB stars is likely similar to that found in the diffuse ISM, making it an unlikely carrier of unidentified depleted oxygen.

Based on observations of the $3.4 \mu \mathrm{m}$ spectral band in the diffuse ISM, at least $3-4 \%$ of interstellar carbon is incorporated in aliphatic hydrocarbons in the refractory organic mantle. These aliphatic hydrocarbons are attached to larger chemical structures, which likely contain up to $10 \%$ of the interstellar carbon (Pendleton \& Allamandola 2002). We find that only about $3 \times 10^{-7}-2 \times 10^{-3}$ of the total $\mathrm{C}$ atoms in the outflow are incorporated into refractory organic material at the end of C-rich outflows. This decreases to $5 \times 10^{-11}-2 \times 10^{-7}$ for O-rich outflows. Together with the low coverage of the AGB dust, it is clear that AGB outflows do not contribute significantly to the refractory organic mantle of interstellar dust.

\subsection{Sub-monolayer grain surface chemistry}

Surface chemistry on dust within AGB outflows generally takes place in the sub-monolayer regime. All outflows have less than one monolayer of ice on the grain surface, except higher density O-rich outflows which are covered by several layers of $\mathrm{H}_{2} \mathrm{O}$ ice (Figs. 4 and 7). Sub-monolayer regimes are not common in astrochemical environments, holding only in the transition region from diffuse to dense interstellar clouds, where ice mantles are formed, and during the desorption of ice mantles at snowlines in protoplanetary disks (Potapov et al. 2019). AGB outflows can now be added to this list.

Compared to dust grains covered with ices, bare dust surfaces can lead to an increased reactivity for reactions involving molecules and/or radicals (Potapov et al. 2020). This surface catalytic effect is likely due to the participation of the dust surface itself in the reactions and an increased diffusion rate (Potapov et al. 2017, 2019; Potapov et al. 2020).

Since the binding energy determines the competition between desorption and diffusion on the grain surface, we have simulated the possible influence of the surface catalytic effect by increasing the binding energies of all ice species in our network by $10 \%$ and $30 \%$. This simplified ap-

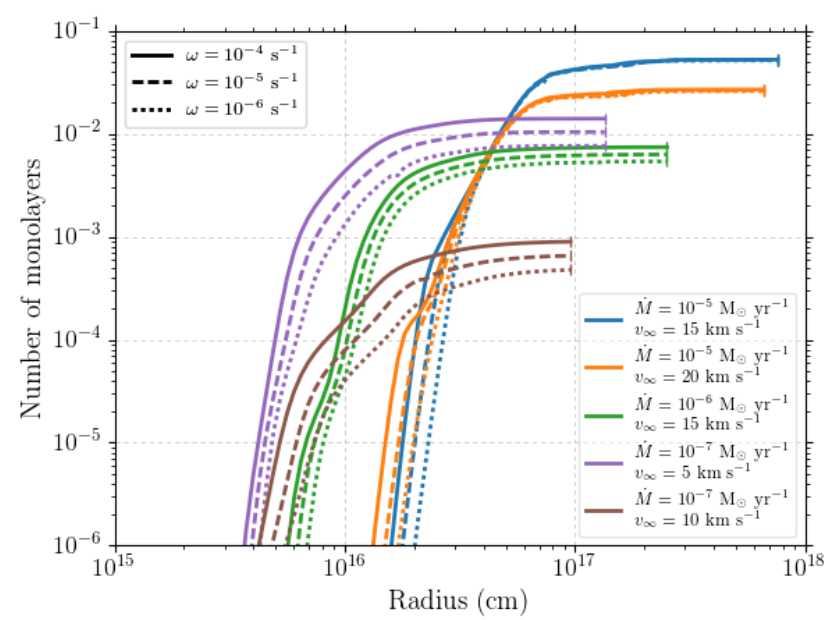

Figure 8. Number of monolayers of refractory organic material in C-rich outflows with different mass-loss rates and outflow velocities, using the parent species of Papers I and II. The different line styles correspond to different values of the photoprocessing rate, $\omega$. The dash marks where the $\mathrm{H}_{2}$ number density reaches 10 $\mathrm{cm}^{-3}$, i.e., where the outflow merges with the ISM.

proach does not take into account the particular dust composition and surface properties or any species-specific increases. Nonetheless, as a first approximation, we find that increasing the binding energy by $10 \%$ and $30 \%$ increases the refractory organic coverage by at least the same factor. The increase is larger for lower density outflows. Compiling surface-dependent binding energies measured in the laboratory would be the next step in assessing the influence of surface catalytic effects.

\subsection{Sensitivity to model parameters}

Several assumptions have been made in order to calculate the observational grids of O-rich and C-rich outflows. Here, we address their possible impact on the formation of refractory organic material.

Photoprocessing rate We assumed a photoprocessing rate of $\omega=10^{-5} \mathrm{~s}^{-1}$, which lies within the range of $3 \times 10^{-4}-8 \times$ $10^{-6} \mathrm{~s}^{-1}$ derived from irradiation times of laboratory experiments (Allamandola et al. 1988; Bernstein et al. 2002). The effect of changing $\omega$ within this range is shown in Fig. 8 for different C-rich outflows, using the parent species of Papers I and II (as in Fig. 4). Only lower density outflows show a significant effect when changing $\omega$. When varying the rate between $10^{-4}$ and $10^{-6} \mathrm{~s}^{-1}$, the refractory organic output in the lowest density outflows varies by a factor of about two. This range decreases with increasing outflow density and becomes negligible for the highest density outflows. As higher density outflows are the main carriers of refractory organic material, changing the photoprocessing rate within the range derived from experiments will not alter our main conclusions.

Grain size distribution The assumed GSD of the dust influences the formation of the ice mantle (Paper II). While assuming that the GSD has been set in the inner wind and 
the grains do not grow throughout the subsequent outflow, a GSD with a larger average grain surface area leads to a larger depletion of gas-phase species and therefore to a thicker ice mantle. Observations of dust grains around AGB stars suggest that AGB dust is large but not single-sized (e.g., Groenewegen 1997; Winters et al. 1997; Hoppe \& Zinner 2000; Khouri et al. 2016; Dell'Agli et al. 2017; Nanni et al. 2018). In Paper II, we found that molecular line observations tentatively suggest an average grain surface area larger than that of the canonical MRN distribution.

A larger average grain surface would lead to a thicker ice mantle and subsequently more refractory organic material. However, because the precise GSD is not yet known, we have assumed the commonly-assumed canonical MRN distribution. In order to quantify the GSD of AGB dust, further observations are necessary as well as a more complete understanding of the dust formation process(es). One key aspect for the formation of refractory organic material is the possible dependence of the GSD on the outflow density.

Drift velocity The drift velocity between dust and gas has a large impact on the accretion of gas-phase species. Higher drift velocities lead to more accretion, and hence to a larger ice mantle, but only up to $v_{\text {drift }} \sim 10 \mathrm{~km} \mathrm{~s}^{-1}$. For larger values, sputtering partly destroys the ice mantle (Paper I).

When modelling the observational grids, we assumed a drift velocity based on the observed mass-loss rate, as higher mass-loss rate outflows generally have lower drift velocities. Using drift velocities specific to the outflow, retrieved through a combination of continuum and line emission radiative transfer, will make the estimate of the refractory organic coverage more accurate.

Spherical symmetry A central assumption to the chemical model is the spherical symmetry of the outflow. However, both large-scale and small-scale deviations from spherical symmetry have been observed, such as spirals (e.g., Mauron \& Huggins 2006; Maercker et al. 2012), equatorial density enhancements (EDEs) or disks (Kervella et al. 2014; Homan et al. 2018) and clumps (e.g. Khouri et al. 2016; Agúndez et al. 2017). The presence of overdensities could lead to an increase in ice formation in these regions. Models specific to such density distributions are necessary to determine whether this leads to a larger refractory organic coverage.

In particular, the presence of an EDE can lead to the overestimation of the mass-loss rate (Decin et al. 2019). Hydrodynamical models suggest that the overestimation can be a factor of a few (El Mellah et al. 2020). A smaller massloss rate would imply a smaller refractory organic coverage. However, the density within EDE can be up to several orders of magnitude larger than that of the surrounding outflow, depending on the intrinsic orbital parameters of the binary system and its inclination. Such an increase in density would have a positive effect on refractory organic formation. To disentangle the effects of the decrease in mass-loss rate and the overdensity within the EDE, specialised (physico)chemical models are needed.

Moreover, large-scale density structures such as EDEs and spirals are likely due to binary interaction of the AGB star with a stellar or planetary companion (e.g., Mauron \& Huggins 2006; Kervella et al. 2014; Ramstedt et al. 2014;
Kim et al. 2015; Decin et al. 2015; Maercker et al. 2016; Decin et al. 2020). If the binary companion has a strong UV field, e.g., a white dwarf, photon-processes are able to occur in the otherwise shielded inner region. Besides the impact on the gas-phase chemistry (Van de Sande \& Millar 2019), this could initiate grain-surface chemistry as well as photoprocessing. The further development of chemical models that account for inner UV photons is necessary.

\section{CONCLUSIONS}

We presented the first observationally motivated study of the refractory organic contribution of AGB outflows to the ISM. The chemical network of Papers I and II was expanded to include a more complex grain surface chemistry, reactive desorption and photodissociation on the grain surface. A new type of reaction was introduced based on laboratory experiments: the photoprocessing of volatile complex ices into inert refractory organic material. This reaction is a sink for complex ices, i.e., ices containing at least three $\mathrm{C}$ atoms or two $\mathrm{C}$ atoms and one other heavy atom. We assembled grids of O- and C-rich outflows from observational samples. New observationally motivated C-rich parent species were also compiled, with one representing an average outflow and the other an outflow with the maximal observed carbon content.

Refractory organic material is able to form on the dust within the outflow. The majority of the material is inherited directly from the gas phase: complex gas-phase species are accreted onto the dust and subsequently photoprocessed into refractory organic material. Grain-surface chemistry plays only a minor role in the formation of complex ice species. This is unlike other astrochemical environments, where reactions between simple ices are crucial to chemical complexity. The amount of refractory organic material formed depends on the outflow density and the initial gas-phase composition. The outflow density plays a twofold role, as it sets the accretion rate and the onset of photon-processes (photodissociation in the gas and on the ice, photodesorption and photoprocessing). Larger density outflows have a larger ice mantle and refractory organic coverage. The initial gasphase composition determines the availability of feedstock ices. The initial abundances of $\mathrm{C}_{2} \mathrm{H}_{2}$ and, to a lesser extent, $\mathrm{HCN}$ are crucial to the refractory organic coverage.

Therefore, dust in O-rich outflows is only negligibly covered by refractory organic material. The average refractory organic coverage is only $0.0001 \%$. In C-rich outflow, the dust has an average refractory organic coverage of $3-9 \%$, depending on the parent species used. This corresponds to only about $10^{-7}-10^{-3}$ of all $\mathrm{C}$ atoms being incorporated into the refractory organic mantle. The coverage increases with increasing outflow density up to $8-22 \%$. However, the small average refractory organic coverage is unlikely to affect the physics and chemistry of the dust in the ISM. The oxygen content of the material agrees with the low $\mathrm{O} / \mathrm{C}$ ratios of $\sim 0.015$ measured in the ISM (Pendleton \& Allamandola 2002).

In order to improve our estimate of the refractory organic contribution to the ISM, measured drift velocities and a better grasp on the GSD of the dust are necessary. These two parameters influence the accretion rate of gas-phase species. Changing the photoprocessing rate within the range 
obtained from laboratory experiments does not significantly affect our results. The grain-surface chemistry takes place in a sub-monolayer regime, which is not common. The surface catalytic effect could increase the refractory organic coverage, but further laboratory experiments using bare dust grains are necessary. Additionally, our spherically symmetric model cannot account for the possible effects of smalland large-scale overdensities within the outflow. For these outflows, models specific to the particular density distribution have to be designed. The impact of a UV-emitting binary companion could also influence the refractory organic output.

This work shows that photoprocessing is a viable chemical process in AGB outflows. Although refractory organic material can be formed in specific outflows, it does not significantly contribute to the refractory organic mantles of interstellar dust. It opens up the question to the refractory organic coverage of other contributors to dust in the ISM, such as supernovae. Characterising pristine stellar dust is not only crucial to understanding its subsequent evolution in the ISM, but also to understanding the origin of refractory organics on interstellar dust.

\section{ACKNOWLEDGEMENTS}

MVdS acknowledges support from the Research Foundation Flanders (FWO) through grant 12X6419N. CW acknowledges financial support from the University of Leeds and from the Science and Technology Facilities Council (grant numbers ST/R000549/1 and ST/T000287/1). TJM thanks STFC for support under grant reference ST/P000312/1.

\section{DATA AVAILABILITY}

The chemical reaction network, chemical kinetics model and all data underlying this article are available on request to the corresponding author.

\section{REFERENCES}

Agúndez M., et al., 2017, A\&A, 601, A4

Agúndez M., Martínez J. I., de Andres P. L., Cernicharo J., Martín-Gago J. A., 2020, A\&A, 637, A59

Allamandola L., Sandford S., Valero G., 1988, Icarus, 76, 225

Bernstein M. P., Dworkin J. P., Sandford S. A., Cooper G. W., Allamandola L. J., 2002, Nature, 416, 401

Bischoff D., Kreuzig C., Haack D., Gundlach B., Blum J., 2020, MNRAS, 497, 2517

Butchart I., McFadzean A. D., Whittet D. C. B., Geballe T. R., Greenberg J. M., 1986, A\&A, 154, L5

Cuppen H. M., Walsh C., Lamberts T., Semenov D., Garrod R. T., Penteado E. M., Ioppolo S., 2017, Space Sci. Rev., 212,1

Danilovich T., et al., 2015, A\&A, 581, A60

Decin L., et al., 2010, A\&A, 516, A69

Decin L., Richards A. M. S., Neufeld D., Steffen W., Melnick G., Lombaert R., 2015, A\&A, 574, A5

Decin L., et al., 2019, Nature Astronomy, 3, 408

Decin L., et al., 2020, Science, 369, 1497

Dell'Agli F., García-Hernández D. A., Schneider R., Ventura P., La Franca F., Valiante R., Marini E., Di Criscienzo M., 2017, MNRAS, 467, 4431
Draine B. T., 2003, ARA\&A, 41, 241

Duley W. W., Scott A. D., Seahra S., Dadswell G., 1998, ApJ, 503, L183

El Mellah I., Bolte J., Decin L., Homan W., Keppens R., 2020, A\&A, 637, A91

Garrod R. T., Widicus Weaver S. L., Herbst E., 2008, ApJ, 682, 283

González Delgado D., Olofsson H., Kerschbaum F., Schöier F. L., Lindqvist M., Groenewegen M. A. T., 2003, A\&A, 411, 123

Greenberg J. M., 1986, Ap\&SS, 128, 17

Greenberg J. M., Shen C., 1999, Ap\&SS, 269, 33

Greenberg J. M., Yencha A. J., Corbett J. W., Frisch H. L., 1972, in Les Spectres des Astres dans l'Infrarouge et les Microondes. pp $425-436$

Groenewegen M. A. T., 1997, A\&A, 317, 503

Hagen W., Allamandola L. J., Greenberg J. M., 1979, Ap\&SS, 65,215

Henning T., Semenov D., 2013, Chemical Reviews, 113, 9016

Heras A. M., Hony S., 2005, A\&A, 439, 171

Höfner S., Olofsson H., 2018, A\&ARv, 26, 1

Homan W., Danilovich T., Decin L., de Koter A., Nuth J., Van de Sande M., 2018, A\&A, 614, A113

Hoppe P., Zinner E., 2000, Journal of Geophysical Research: Space Physics, 105, 10371

Jäger C., Dorschner J., Mutschke H., Posch T., Henning T., 2003, A\&A, 408, 193

Jones A. P., Fanciullo L., Köhler M., Verstraete L., Guillet V., Bocchio M., Ysard N., 2013, A\&A, 558, A62

Kervella P., et al., 2014, A\&A, 564, A88

Khouri T., et al., 2016, A\&A, 591, A70

Kim H., et al., 2015, ApJ, 814, 61

Kouchi A., Kudo T., Nakano H., Arakawa M., Watanabe N., Sirono S.-i., Higa M., Maeno N., 2002, ApJ, 566, L121

Maercker M., et al., 2012, Nature, 490, 232

Maercker M., Danilovich T., Olofsson H., De Beck E., Justtanont K., Lombaert R., Royer P., 2016, A\&A, 591, A44

Materese C. K., Nuevo M., Sandford S. A., 2017, Astrobiology, 17,761

Mathis J. S., 1979, ApJ, 232, 747

Mathis J. S., Rumpl W., Nordsieck K. H., 1977, ApJ, 217, 425

Mauron N., Huggins P. J., 2006, A\&A, 452, 257

McElroy D., Walsh C., Markwick A. J., Cordiner M. A., Smith K., Millar T. J., 2013, A\&A, 550, A36

Millar T. J., Herbst E., 1994, A\&A, 288, 561

Min M., Dullemond C. P., Dominik C., de Koter A., Hovenier J. W., 2009, A\&A, 497, 155

Muñoz Caro G. M., Schutte W. A., 2003, A\&A, 412, 121

Mutschke H., Begemann B., Dorschner J., Guertler J., Gustafson B., Henning T., Stognienko R., 1998, A\&A, 333, 188

Nanni A., Marigo P., Girardi L., Rubele S., Bressan A., Groenewegen M. A. T., Pastorelli G., Aringer B., 2018, MNRAS, 473,5492

Olofsson H., González Delgado D., Kerschbaum F., Schöier F. L., 2002, A\&A, 391, 1053

Pendleton Y. J., Allamandola L. J., 2002, ApJS, 138, 75

Potapov A., Jäger C., Henning T., Jonusas M., Krim L., 2017, The Astrophysical Journal, 846, 131

Potapov A., Theulé P., Jäger C., Henning T., 2019, The Astrophysical Journal, 878, L20

Potapov A., Jäger C., Henning T., 2020, arXiv e-prints, p. arXiv:2005.00757

Preibisch T., Ossenkopf V., Yorke H. W., Henning T., 1993, A\&A, 279,577

Ramstedt S., Schöier F. L., Olofsson H., Lundgren A. A., 2008, A\&A, 487, 645

Ramstedt S., et al., 2014, A\&A, 570, L14

Rau G., Hron J., Paladini C., Aringer B., Eriksson K., Marigo P., Nowotny W., Grellmann R., 2017, A\&A, 600, A92 
Sandford S. A., Allamandola L. J., Tielens A. G. G. M., Sellgren K., Tapia M., Pendleton Y., 1991, ApJ, 371, 607

Schöier F. L., Olofsson H., 2001, A\&A, 368, 969

Tielens A. G. G. M., 2005, The Physics and Chemistry of the Interstellar Medium. Cambridge University Press

Tielens A. G. G. M., 2013, Rev. Mod. Phys., 85, 1021

Van de Sande M., Millar T. J., 2019, ApJ, 873, 36

Van de Sande M., Decin L., Lombaert R., Khouri T., de Koter A., Wyrowski F., De Nutte R., Homan W., 2018, A\&A, 609, A63

Van de Sande M., Walsh C., Mangan T. P., Decin L., 2019, MNRAS, p. 2325

Van de Sande M., Walsh C., Danilovich T., 2020, MNRAS, 495, 1650

Walsh C., Millar T. J., Nomura H., Herbst E., Widicus Weaver S., Aikawa Y., Laas J. C., Vasyunin A. I., 2014, A\&A, 563, A33

Whittet D. C. B., 2010, ApJ, 710, 1009

Willner S. P., Russell R. W., Puetter R. C., Soifer B. T., Harvey P. M., 1979, ApJ, 229, L65

Winters J. M., Fleischer A. J., Le Bertre T., Sedlmayr E., 1997, A\&A, 326, 305

Yang X., Chen P., He J., 2004, A\&A, 414, 1049

Zhukovska S., Henning T., 2013, A\&A, 555, A99

\section{APPENDIX A: DETAILS OF THE CHEMICAL KINETICS MODEL}

The chemical kinetics model is based on that of Papers I and II, and originates from the publicly available UMIST Database for Astrochemistry (UDfA) circumstellar envelope (CSE) model (McElroy et al. 2013) ${ }^{1}$. The model uniquely describes both gas-phase and dust-gas chemistry: gas-phase species are able to accrete onto the dust (forming an ice mantle), react on the dust surface through the reactive Langmuir-Hinshelwood and the stick-and-hit Eley-Rideal mechanisms, and return to the gas phase via thermal desorption, photodesorption and sputtering (destroying the ice mantle). The ice mantle is assumed to be physisorbed onto the dust, and not chemically integrated within the dust. A complete description of the dust-gas chemistry and its reaction rates is given in Paper I.

The one-dimensional model describes a uniformly expanding spherically symmetric outflow, with constant massloss rate, $\dot{M}$, and gas outflow velocity, $v_{\infty}$. The gas temperature profile is described by a power law,

$T_{\text {gas }}(r)=T_{*}\left(\frac{r}{R_{*}}\right)^{-\epsilon}$,

where $T_{*}$ and $R_{*}$ are the stellar temperature and radius. Dust is assumed to be present at the start of the model, at $10^{15} \mathrm{~cm} \approx 20 \mathrm{R}_{*}$. Therefore, we assume that dust formation occurs in the inner wind and dust is injected into the intermediate wind with a certain grain size distribution (GSD) that does not change throughout the outflow. In this paper, the GSD is fixed to the canonical value of (Mathis et al. 1977, MRN). The dust is assumed to have a fixed drift velocity, $v_{\text {drift }}$, relative to the gas. The dust temperature profile is

1 http://udfa.ajmarkwick.net/index.php?mode=downloads

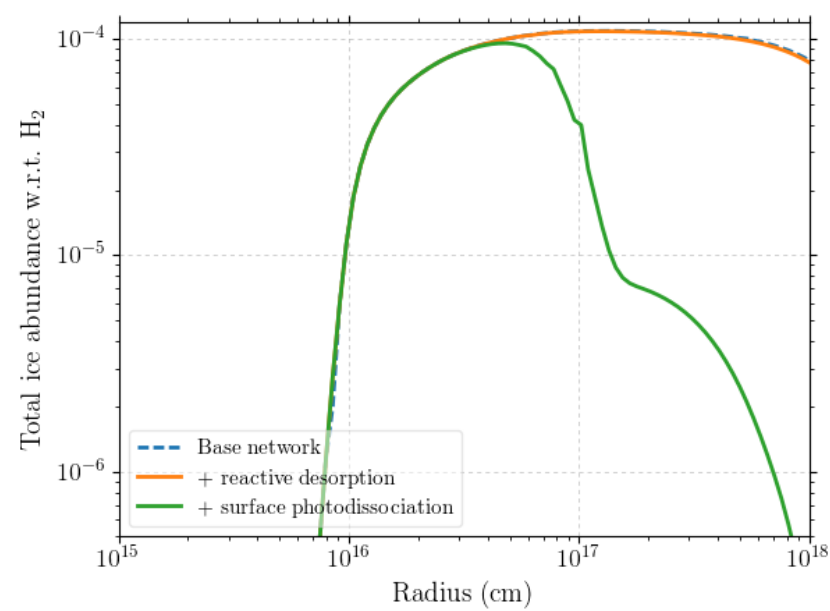

Figure B1. Total ice abundance relative to $\mathrm{H}_{2}$ in an O-rich outflow with $\dot{M}=10^{-5} \mathrm{M}_{\odot} \mathrm{yr}^{-1}, v_{\infty}=15 \mathrm{~km} \mathrm{~s}^{-1}$ and $v_{\text {drift }}=5 \mathrm{~km}$ $\mathrm{s}^{-1}$. Blue dashed line: results from the updated chemical network. Orange solid line: updated network + reactive desorption. Green solid line: updated network + reactive desorption + grain surface photodissociation.

described by

$T_{\text {dust }}(r)=T_{\text {dust }, *}\left(\frac{2 r}{R_{*}}\right)^{-\frac{2}{4+s}}$,

where $T_{\text {dust,* }}$ and $s$ are free parameters. Their values depend on the mass-loss rate, outflow velocity, and the composition of the dust. To derive them, we use the continuum radiative transfer code MCMAx (see also Appendix C).

\section{APPENDIX B: INFLUENCE OF THE NEW CHEMICAL NETWORK ON THE GAS AND ICE PHASES}

In this Appendix, we show the influence of including reactive desorption and grain surface photodissociation to the chemical reaction network. We find that reactive desorption alone does not have a large influence on the total ice abundance throughout the outflow. Together with grain-surface photodissociation, however, these two new reactions have a significant effect on the ice mantle. Grain-surface photodissociation leads to more radical ice species, which can react via the Langmuir-Hinshelwood mechanism and subsequently desorb from the grain. Additionally, the photodissociation products can be thermally desorbed if their binding energy is lower. Since the ices are destroyed by photons, rather than photodesorbed in their entirety, the gas-phase abundance of the species will be lower, which leads to less reaccretion.

Sects. B1 and B2 describe the effects of the two new reactions on the ice component throughout an O-rich and a C-rich outflow, respectively. In our notation, the prefix ' $G$ ' (grain) denotes ice species.

\section{B1 O-rich outflow}

Fig. $\mathrm{B} 1$ shows the total ice abundance relative to $\mathrm{H}_{2}$ in an $\mathrm{O}-$ rich outflow, characterised by $\dot{M}=10^{-5} \mathrm{M}_{\odot} \mathrm{yr}^{-1}, v_{\infty}=15$ $\mathrm{km} \mathrm{s}^{-1}$ and $v_{\text {drift }}=5 \mathrm{~km} \mathrm{~s}^{-1}$ for different chemical networks. 


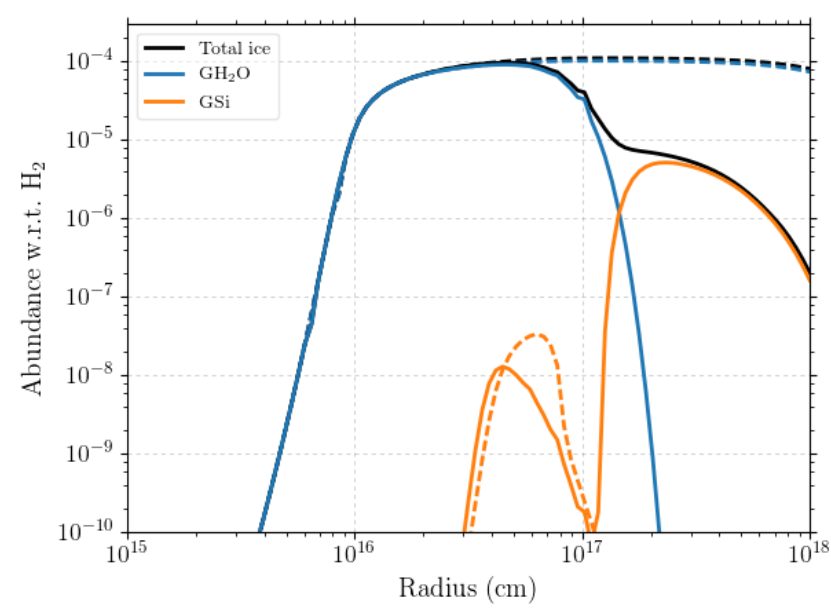

Figure B2. Fractional abundance of key ice species in an O-rich outflow with $\dot{M}=10^{-5} \mathrm{M}_{\odot} \mathrm{yr}^{-1}, v_{\infty}=15 \mathrm{~km} \mathrm{~s}^{-1}$ and $v_{\text {drift }}=5$ $\mathrm{km} \mathrm{s}^{-1}$. Dashed lines: results from the updated chemical network. Solid lines: results from the updated network + reactive desorption + grain surface photodissociation. The total fractional ice abundance is plotted in black.

We show the results using the base chemical network, which includes all reactions described in Papers I and II, the base network including reactive desorption, and the base network including reactive desorption and grain-surface photodissociation. Fig. B2 shows the fractional abundances of the main ice components for the base network and the network including both reactions.

Including reactive desorption has a very small effect on the total ice abundance in the outer region of the outflow, where the ice abundance of radicals increases due to accretion only in this network. When using the base network and the network including reactive desorption, the main ice component is $\mathrm{GH}_{2} \mathrm{O}$ throughout the outflow. When including grain-surface photodissociation, $\mathrm{GH}_{2} \mathrm{O}$ is broken up, providing $\mathrm{GOH}$ radicals. Two of these radicals can react, forming $\mathrm{GH}_{2} \mathrm{O}_{2}$, which subsequently desorbs upon reaction. This removes water both from the ice and the gas phase, hindering its reaccreation. The gas-phase abundance of $\mathrm{H}_{2} \mathrm{O}_{2}$ also increases. For this dense outflow, we find that its peak abundance around $10^{17} \mathrm{~cm}$ (i.e., before the onset of photodissociation) increases from $\sim 10^{-10}$ to $\sim 10^{-5}$ relative to $\mathrm{H}_{2}$. The peak abundance decreases to $\sim 10^{-7}$ relative to $\mathrm{H}_{2}$ for lower density outflows.

After the removal of $\mathrm{GH}_{2} \mathrm{O}$, the main ice component depends on the outflow density. In high-density outflows, the main ice component is GSi. This is the result of grain surface photodissociation of GSiO, and to a lesser extent GSiS. While GO is very reactive, GSi remains on the grain. For outflows with $\dot{M}<10^{-5} \mathrm{M}_{\odot} \mathrm{yr}^{-1}$, the main component is $\mathrm{GSiH}_{4}$ due to a larger $\mathrm{GH}$ abundance thanks to the onset of photon-processes closer to the star. This leads to an increase in its gas-phase abundance. The peak gas-phase abundance of $\mathrm{SiH}_{4}$ ranges with increasing outflow density from $\sim 10^{-12}$ to $\sim 10^{-8}$ relative to $\mathrm{H}_{2}$.

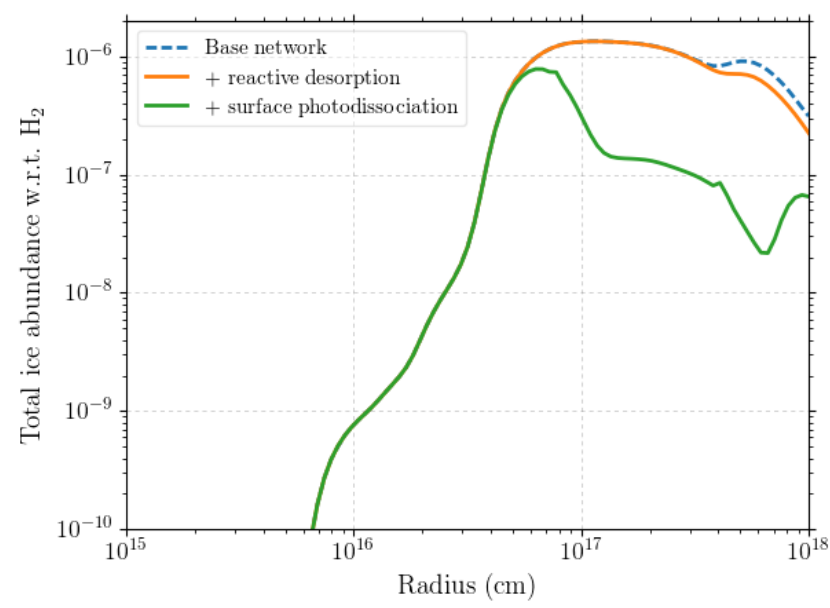

Figure B3. Total ice abundance relative to $\mathrm{H}_{2}$ in an C-rich outflow with $\dot{M}=10^{-5} \mathrm{M}_{\odot} \mathrm{yr}^{-1}, v_{\infty}=15 \mathrm{~km} \mathrm{~s}^{-1}$ and $v_{\text {drift }}=5 \mathrm{~km}$ $\mathrm{s}^{-1}$, using the parents species used in Papers I and II. Blue dashed line: results from the updated chemical network. Orange solid line: updated network + reactive desorption. Green solid line: updated network + reactive desorption + grain surface photodissociation.

\section{B2 C-rich outflow}

Similar to the O-rich outflow, Fig. B3 shows the total ice abundance relative to $\mathrm{H}_{2}$ in a C-rich outflow, characterised by $\dot{M}=10^{-5} \mathrm{M}_{\odot} \mathrm{yr}^{-1}, v_{\infty}=15 \mathrm{~km} \mathrm{~s}^{-1}$ and $v_{\text {drift }}=5$ $\mathrm{km} \mathrm{s}^{-1}$ for different chemical networks, using the parents species used in Papers I and II. Fig. B4 shows the fractional abundances of its main ice components for the base network and the network including both reactions.

Including reactive desorption again only has a small effect on the total ice abundance in the outer region of the outflow. Using the base model and the model including reactive desorption, the main ice components are $\mathrm{GHCN}$ and $\mathrm{GH}_{2} \mathrm{O}$. The bump in the ice abundance in the outer outflow is due to $\mathrm{GH}_{2} \mathrm{O}$. It is not present when using the chemical network of Papers I and II and is formed thanks to the more complex surface chemical network. When including grain surface photodissociation, GHCN and $\mathrm{GH}_{2} \mathrm{O}$ are photodissociated, yielding $\mathrm{GOH}+\mathrm{GOH}$ (which reactively desorbs, see Sect. B1) and GH + GCN. The peak in GNO at the end of the outflow is linked to the surface photodissociation of GHCN, via the reaction sequence

$\mathrm{GO}+\mathrm{GCN} \rightarrow \mathrm{GOCN}$,

$\mathrm{GH}+\mathrm{GOCN} \rightarrow \mathrm{GHOCN}$,

$\mathrm{GHOCN}+h \nu \rightarrow \mathrm{GOH}+\mathrm{GNH}$,

$\mathrm{GO}+\mathrm{GNH} \rightarrow \mathrm{GHNO}$,

$\mathrm{GO}+\mathrm{GHNO} \rightarrow \mathrm{GNO}+\mathrm{GOH}$.

\section{APPENDIX C: PARAMETERS OF THE OBSERVATIONAL GRIDS OF OUTFLOWS}

Tables C.1 and C.2 list the observational set of C-rich and Orich outflows, respectively, with the observed mass-loss rates and outflow velocities and the assumed drift velocities. As in Papers I and II, the parameters $T_{\text {dust,* }}$ and $s$ of the dust 


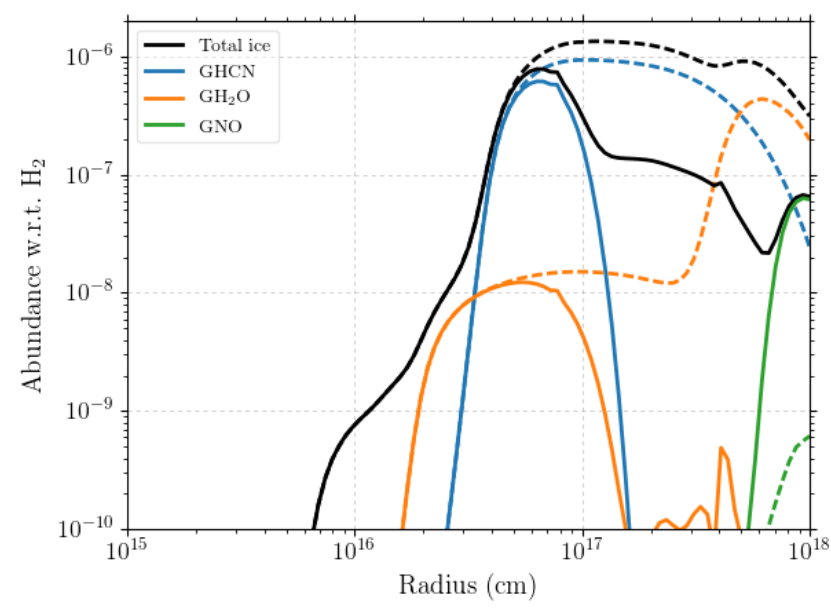

Figure B4. Fractional abundances of key ice species in an Crich outflow with $\dot{M}=10^{-5} \mathrm{M}_{\odot} \mathrm{yr}^{-1}, v_{\infty}=15 \mathrm{~km} \mathrm{~s}^{-1}$, and $v_{\text {drift }}=5 \mathrm{~km} \mathrm{~s}^{-1}$, using the parents species used in Papers I and II. Dashed lines: results from the updated chemical network. Solid lines: results from the updated network + reactive desorption + grain surface photodissociation. The total fractional ice abundance is plotted in black.

temperature power-law are obtained by fitting Eq. A2 to the dust temperature profiles calculated using the continuum radiative transfer code MCMAX (Min et al. 2009). This was done by identifying the minimum value of the $\chi^{2}$ test when varying $T_{\text {dust,* }}$ in intervals of $50 \mathrm{~K}$ and $s$ in intervals of 0.1 . The minimum $\chi^{2}$ values of the fitting routine are also included in Tables 3 and 4 . The optical constants used in the radiative transfer modelling for the O-rich outflows are those of Mutschke et al. (1998) for melilite and those of Jäger et al. (2003) for silicate with Fe. For the C-rich outflows, we used the optical constants of Preibisch et al. (1993) for amorphous carbon.

\section{APPENDIX D: INFLUENCE ON THE GAS-PHASE COMPOSITION}

The influence on the gas-phase composition is the main topic of Paper I, where we found that including dust-gas chemistry can significantly influence the gas phase throughout the outflow. The depletion of parent species depends on the outflow density, the drift velocity, the dust temperature as well as the GSD (Paper II).

Consistent with the results of Papers I and II, the depletion of parent species is smaller than one order of magnitude for both observational sets of outflows. This is in part due to the low drift velocities assumed for the highest mass-loss rates, the assumption of the canonical MRN GSD as well as the warmer 50/50 mixture of melilite and silicate with $\mathrm{Fe}$ dust used in the O-rich calculations.

Daughter species can be affected by dust-gas chemistry in two ways. They can be depleted onto the dust, which occurs more efficiently for species with a large binding energy, or experience an increase in abundance because of their formation on the dust, followed by thermal desorption, photodesorption or sputtering. Because of the more com-

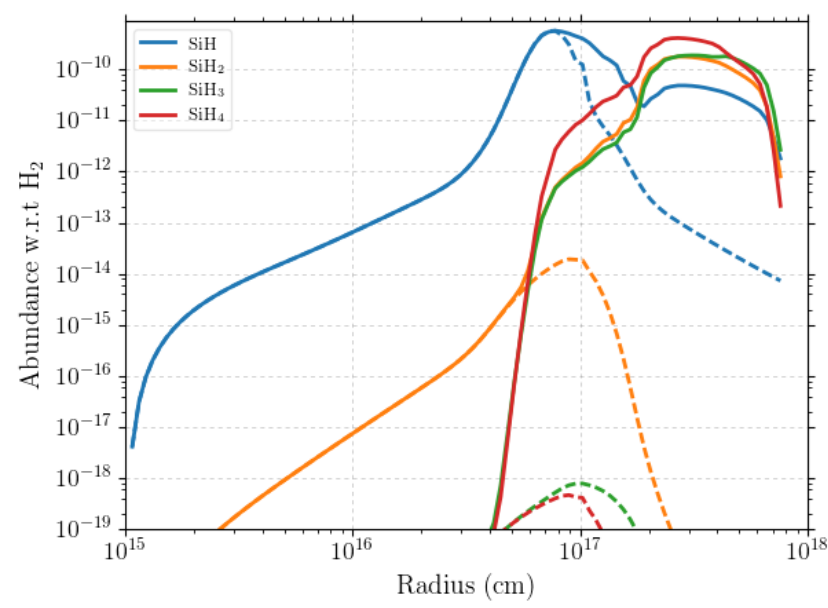

Figure D1. Abundance profiles of daughter species produced by grain-surface chemistry in a C-rich outflow with $\dot{M}=10^{-5} \mathrm{M}_{\odot}$ $\mathrm{yr}^{-1}, v_{\infty}=15 \mathrm{~km} \mathrm{~s}^{-1}$ and $v_{\text {drift }}=5 \mathrm{~km} \mathrm{~s}^{-1}$, using the parent species of Paper I. Solid lines: results obtained when including the full chemical network. Dashed lines: results obtained without including dust-gas chemistry.

plex grain-surface chemical network, the different species can form on the grain surface, leading to a different gas-phase return compared to Paper I.

Figs. D1 and D2 show gas-phase abundance profiles of daughter species produced on grain surfaces for a C-rich and O-rich outflow, respectively. Hydrates are efficiently formed on the grain surface thanks to the high mobility of $\mathrm{H}$ and are subsequently thermally desorbed to the gas phase. The largest influence is seen for $\mathrm{SiH}_{n}(n=1,2,3,4)$ in both Crich and O-rich outflows. Reactions with other mobile atoms, such as $\mathrm{O}$, also lead to gas-phase increases (e.g., $\mathrm{NO}_{2}$ in $\mathrm{O}-$ rich outflows). As mentioned in Appendix $\mathrm{B}, \mathrm{H}_{2} \mathrm{O}_{2}$ is released into the gas phase via reactive desorption in O-rich outflows.

Thanks to the complex grain-surface chemical network, various complex ices can be formed on the grain and are released to the gas phase via reactive desorption. Although this increases the gas-phase abundances of simple and complex organic molecules, with surface reactions being the only formation route for some (e.g., $\mathrm{CH}_{3} \mathrm{COOH}$ ), their peak gasphase abundances and column densities remain low. Not including photoprocessing increases their gas-phase abundances, but does not lead to detectable peak gas-phase abundances or column densities. The influence on the gas-phase abundance of C-bearing species is therefore limited due to the extended chemical network, as it distributes the total carbon budget over many species.

\section{APPENDIX E: REFRACTORY ORGANIC OUTPUT OF O-RICH OUTFLOWS}

Fig. E1 shows the refractory organic coverage of the dust in the O-rich observational grid. The average refractory organic output to the ISM is listed in Table 4. The refractory organic coverage again increases with increasing outflow density. Dust grains in O-rich outflows have a refractory organic 


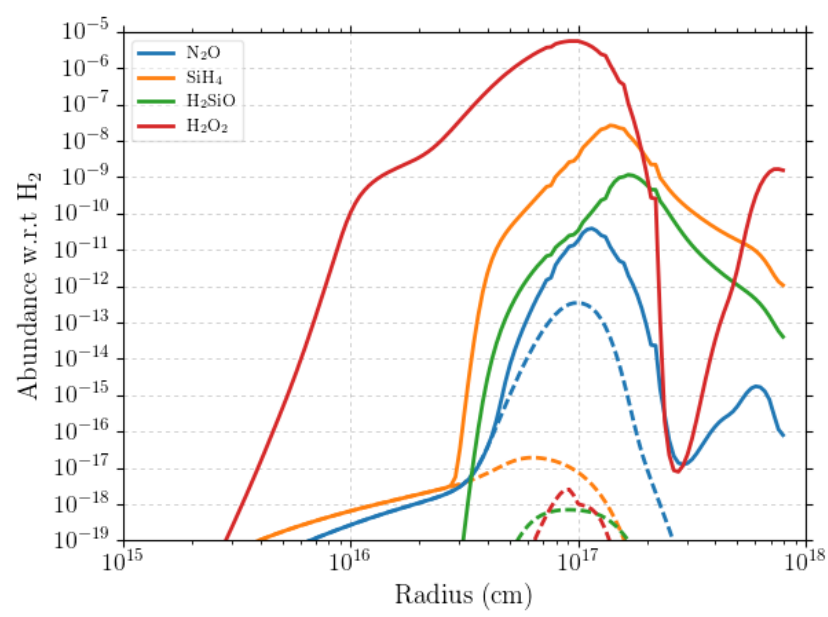

Figure D2. Abundance profiles of daughter species produced by grain-surface chemistry in a O-rich outflow with $\dot{M}=10^{-5} \mathrm{M}_{\odot}$ $\mathrm{yr}^{-1}, v_{\infty}=15 \mathrm{~km} \mathrm{~s}^{-1}$ and $v_{\text {drift }}=5 \mathrm{~km} \mathrm{~s}^{-1}$. Solid lines: results obtained when including the full chemical network. Dashed lines: results obtained without including dust-gas chemistry.

coverage about four orders of magnitude smaller compared to C-rich outflows.

Fig. E2 shows the fractional composition of the refractory organic material. The same processes are at play as in the C-rich outflows: accretion of gas-phase daughter species followed by the onset of photon-reactions, where the onset depends on the outflow density. We find that the same trends with increasing outflow density are present for the O-rich and the C-rich outflows (Sect. 3.1 and Fig. 4.1), except for the H-bearing component.

In the O-rich outflows, the fraction of H-bearing species decreases with increasing outflow density. C-bearing daughter species are formed in the gas phase, though with much smaller abundances than in C-rich outflows. These are accreted more efficiently on the colder dust and photoprocessed closer to the star in lower density outflows, leading to larger component C-chains, part of which are H-bearing, in the ice and refractory organic material. To a lesser extent, the decreasing fraction of H-bearing species follows the increased fraction of O-bearing species. Because of the larger $\mathrm{H}_{2} \mathrm{O}$ abundance, the amount of O-bearing radicals on the grain is larger, leading to a larger refractory organic component that increases with outflow density. The main Obearing components do not contain any $\mathrm{H}\left(\mathrm{GC}_{n} \mathrm{O}, n=2,3\right)$.

The reversal in the trends for outflows with $\dot{M}>10^{-5}$ $\mathrm{M}_{\odot} \mathrm{yr}^{-1}$ is due to the later onset of $\mathrm{CO}$ and $\mathrm{HCN}$ photodissociation, which shifts to the outer edge of the wind within these outflows. The larger $\mathrm{CO}$ envelope leads to a larger GCO abundance, which is also less likely to be photodissociated into GC and GO. A larger GCO abundance leads to a larger $\mathrm{GC}_{n} \mathrm{O}$ abundance via reactions with $\mathrm{GC}$, increasing the fraction of O-bearing species in the refractory organic mantle. The larger GHCN abundance is less likely to be photodissociated into GH and GCN. The latter is the main reactant involved in the formation of the $\mathrm{N}$-bearing component.

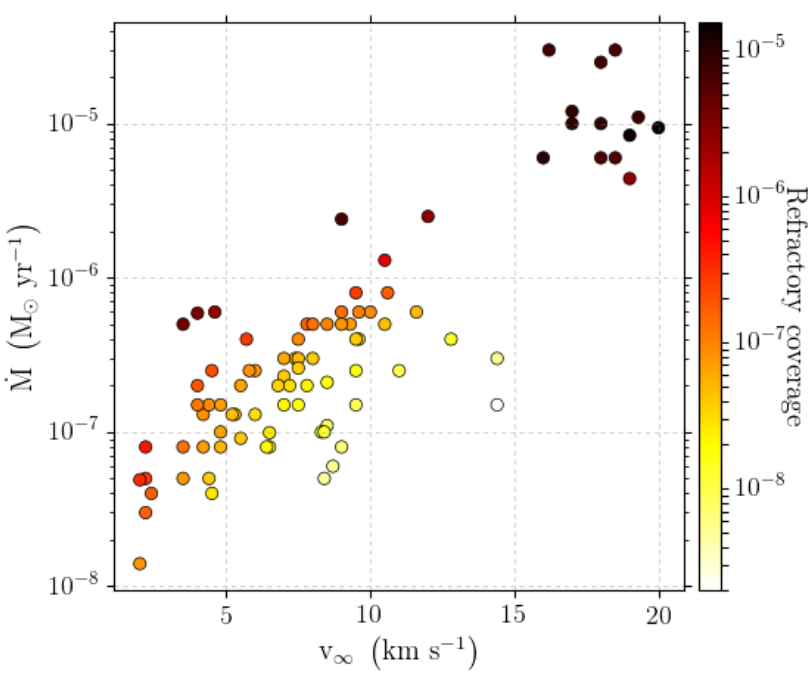

Figure E1. Refractory organic coverage of the dust grains in the outflows of the O-rich observational grid. The coverage corresponds to the number of refractory organic monolayers where the outflow merges with the ISM, which is taken to be the radius where $n\left(\mathrm{H}_{2}\right) \leq 10 \mathrm{~cm}^{-3}$.

This paper has been typeset from a $\mathrm{T}_{\mathrm{E}} \mathrm{X} / \mathrm{L} \mathrm{T}_{\mathrm{E}} \mathrm{X}$ file prepared by the author. 

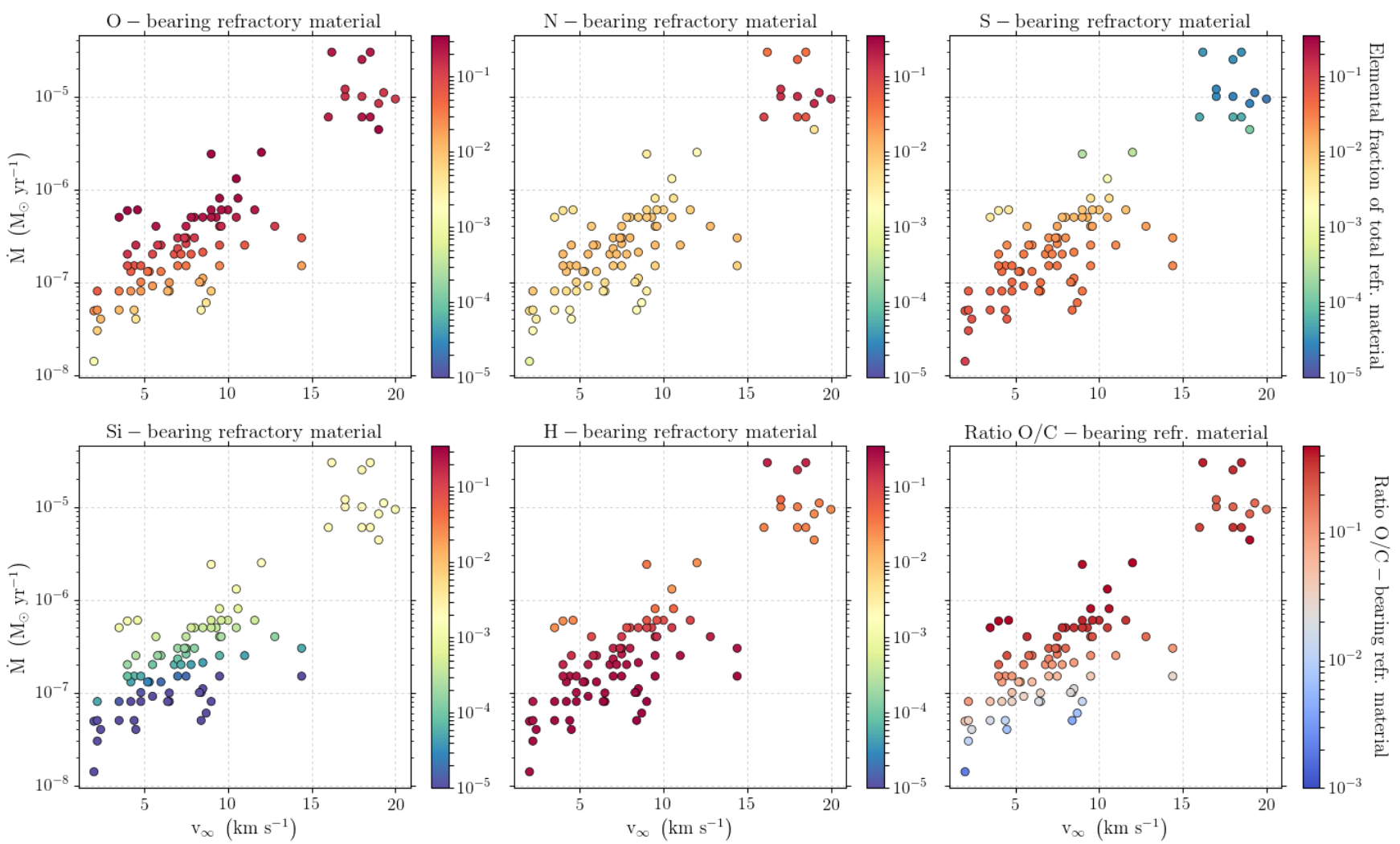

Figure E2. First five panels: elemental fractional composition of the total refractory organic output (O-, N-, S-, Si-, and H-bearing refractory organic material) for the outflows of the O-rich observational dataset. Lower right panel: ratio of O-bearing/C-bearing refractory organic material of the total refractory organic output. 
Table C.1. Observational set of C-rich outflows with their observed mass-loss rates, $\dot{M}\left[\mathrm{M}_{\odot} \mathrm{yr}^{-1}\right]$, and expansion velocities, $v_{\infty}\left[\mathrm{km} \mathrm{s}^{-1}\right]$, from Schöier \& Olofsson (2001) and Danilovich et al. (2015) and the assumed drift velocities, $v_{\text {drift }}\left[\mathrm{km} \mathrm{s}^{-1}\right]$. The values of the free parameters of the dust temperature profile $T_{\mathrm{dust}, *}$ and $s$, together with the chi square value of each fit, are also listed.

\begin{tabular}{|c|c|c|c|c|c|c|c|c|c|c|c|c|c|}
\hline Name & $\dot{M}$ & $v_{\infty}$ & $v_{\text {drift }}$ & $T_{\text {dust }, *}$ & $s$ & $\chi^{2}$ & Name & $\dot{M}$ & $v_{\infty}$ & $v_{\text {drift }}$ & $T_{\text {dust }, *}$ & $s$ & $\chi^{2}$ \\
\hline HD 124268 & $1 \mathrm{e}-07$ & 11.0 & 15.0 & 2050 & 0.8 & 0.35 & SS Vir & $2 \mathrm{e}-07$ & 12.5 & 10.0 & 2000 & 0.8 & 0.23 \\
\hline CZ Hya & $9 e-07$ & 12.0 & 10.0 & 1850 & 0.8 & 1.87 & Y Pav & $1.6 \mathrm{e}-07$ & 8.0 & 10.0 & 2000 & 0.8 & 3.3 \\
\hline V460 Cyg & $1.8 \mathrm{e}-07$ & 10.0 & 10.0 & 2000 & 0.8 & 1.82 & U Hya & $8.9 \mathrm{e}-08$ & 6.5 & 15.0 & 2000 & 0.8 & 1.47 \\
\hline X Vel & $1.8 \mathrm{e}-07$ & 10.0 & 10.0 & 2000 & 0.8 & 1.82 & TW Oph & $5 e-08$ & 7.5 & 15.0 & 2050 & 0.8 & 1.54 \\
\hline BL Ori & $1.1 \mathrm{e}-07$ & 9.0 & 10.0 & 2000 & 0.8 & 3.74 & XZ Vel & $6 \mathrm{e}-07$ & 14.0 & 10.0 & 1900 & 0.8 & 0.47 \\
\hline UU Aur & $1.7 \mathrm{e}-07$ & 12.0 & 10.0 & 2000 & 0.8 & 1.14 & TU Gem & $2.5 \mathrm{e}-07$ & 11.5 & 10.0 & 1950 & 0.8 & 2.54 \\
\hline Y Hya & $1.9 \mathrm{e}-07$ & 9.0 & 10.0 & 1950 & 0.8 & 4.5 & CL Mon & $2.2 \mathrm{e}-06$ & 25.0 & 10.0 & 1800 & 0.8 & 3.28 \\
\hline GY Cam & $3.7 \mathrm{e}-06$ & 20.0 & 10.0 & 1750 & 0.8 & 2.38 & V1426 Cyg & $1 \mathrm{e}-05$ & 14.0 & 5.0 & 1800 & 0.6 & 5.37 \\
\hline RV Cyg & $4.5 \mathrm{e}-07$ & 13.5 & 10.0 & 1900 & 0.8 & 4.92 & CCS 2792 & $6.5 \mathrm{e}-07$ & 17.0 & 10.0 & 1900 & 0.8 & 0.7 \\
\hline HV Cas & $9 \mathrm{e}-07$ & 18.5 & 10.0 & 1900 & 0.8 & 3.06 & II Lup & $1.7 \mathrm{e}-05$ & 21.5 & 5.0 & 1800 & 0.6 & 4.85 \\
\hline TT Tau & $7 \mathrm{e}-08$ & 5.0 & 15.0 & 2000 & 0.8 & 1.25 & V1968 Cyg & $7.5 \mathrm{e}-06$ & 20.0 & 10.0 & 1750 & 0.7 & 2.57 \\
\hline V384 Per & $2.6 \mathrm{e}-06$ & 15.0 & 10.0 & 1750 & 0.8 & 2.16 & RW Lmi & $6 e-06$ & 17.0 & 10.0 & 1750 & 0.7 & 3.13 \\
\hline U Cyg & $9 \mathrm{e}-07$ & 13.0 & 10.0 & 1850 & 0.8 & 0.85 & Y Cvn & $1.5 \mathrm{e}-07$ & 8.5 & 10.0 & 2000 & 0.8 & 0.99 \\
\hline $\mathrm{V}$ Aql & $1.3 \mathrm{e}-07$ & 11.0 & 10.0 & 2000 & 0.8 & 3.66 & RV Aqr & $2.3 \mathrm{e}-06$ & 15.0 & 10.0 & 1850 & 0.7 & 1.56 \\
\hline T Dra & $1.2 \mathrm{e}-06$ & 13.5 & 10.0 & 1800 & 0.8 & 3.13 & CW Leo & $1.5 \mathrm{e}-05$ & 14.5 & 5.0 & 1800 & 0.6 & 9.14 \\
\hline W Cma & $3 e-07$ & 10.5 & 10.0 & 1950 & 0.8 & 0.38 & $\mathrm{X}$ Cnc & $1 \mathrm{e}-07$ & 7.0 & 15.0 & 2000 & 0.8 & 0.87 \\
\hline V688 Mon & $6.1 \mathrm{e}-06$ & 13.5 & 10.0 & 1750 & 0.7 & 1.55 & W Pic & $3 e-07$ & 16.0 & 10.0 & 2000 & 0.8 & 1.81 \\
\hline V821 Her & $3 e-06$ & 13.5 & 10.0 & 1800 & 0.7 & 1.88 & V701 Cas & $4.5 \mathrm{e}-06$ & 11.5 & 10.0 & 1750 & 0.7 & 2.37 \\
\hline IRC +60041 & $3 e-06$ & 28.0 & 10.0 & 1800 & 0.8 & 1.24 & $\mathrm{~T}$ Ind & $9 \mathrm{e}-08$ & 6.0 & 15.0 & 2000 & 0.8 & 0.48 \\
\hline RT Cap & $1 \mathrm{e}-07$ & 8.0 & 15.0 & 2000 & 0.8 & 3.0 & AQ Sgr & $2.5 \mathrm{e}-07$ & 10.0 & 10.0 & 1950 & 0.8 & 0.23 \\
\hline S Aur & $2.2 \mathrm{e}-06$ & 25.5 & 10.0 & 1800 & 0.8 & 2.56 & R Lep & $8.7 \mathrm{e}-06$ & 18.0 & 10.0 & 1750 & 0.7 & 2.14 \\
\hline WZ Cas & $6.5 \mathrm{e}-09$ & 2.5 & 15.0 & 2100 & 0.8 & 0.46 & V466 Per & $1.3 \mathrm{e}-07$ & 9.0 & 10.0 & 2000 & 0.8 & 0.87 \\
\hline R For & $1.3 \mathrm{e}-06$ & 16.5 & 10.0 & 1850 & 0.8 & 5.08 & W Ori & $7 \mathrm{e}-08$ & 11.0 & 15.0 & 2050 & 0.8 & 1.81 \\
\hline Y Tau & $4 e-07$ & 11.0 & 10.0 & 1900 & 0.8 & 2.79 & SZ Car & $2 \mathrm{e}-06$ & 14.0 & 10.0 & 1850 & 0.7 & 1.95 \\
\hline $\mathrm{X} \operatorname{Tr} \mathrm{A}$ & $1.9 \mathrm{e}-07$ & 6.5 & 10.0 & 1950 & 0.8 & 1.66 & V1259 Ori & $8.8 \mathrm{e}-06$ & 16.0 & 10.0 & 1750 & 0.7 & 5.01 \\
\hline RY Mon & $3.5 \mathrm{e}-07$ & 11.0 & 10.0 & 1950 & 0.8 & 3.54 & ST Cam & $9 \mathrm{e}-08$ & 9.0 & 15.0 & 2050 & 0.8 & 1.7 \\
\hline AI Vol & $4.9 \mathrm{e}-06$ & 12.0 & 10.0 & 1750 & 0.7 & 1.82 & Z Psc & $2.5 \mathrm{e}-08$ & 3.5 & 15.0 & 2050 & 0.8 & 0.87 \\
\hline S Cep & $1.5 \mathrm{e}-06$ & 22.0 & 10.0 & 1850 & 0.8 & 1.04 & V Cyg & $1.2 \mathrm{e}-06$ & 11.5 & 10.0 & 1800 & 0.8 & 1.26 \\
\hline R Vol & $1.8 \mathrm{e}-06$ & 18.0 & 10.0 & 1800 & 0.8 & 1.1 & HD 121658 & $1 \mathrm{e}-07$ & 6.5 & 15.0 & 2000 & 0.8 & 0.38 \\
\hline LP And & $1.5 \mathrm{e}-05$ & 14.0 & 5.0 & 1800 & 0.6 & 10.05 & TW Hor & $9 \mathrm{e}-08$ & 5.5 & 15.0 & 2000 & 0.8 & 0.36 \\
\hline V CrB & $3.3 \mathrm{e}-07$ & 7.5 & 10.0 & 1900 & 0.8 & 1.11 & VX And & $4 \mathrm{e}-08$ & 11.5 & 15.0 & 2100 & 0.8 & 0.47 \\
\hline PQ Cep & $1.4 \mathrm{e}-06$ & 19.5 & 10.0 & 1850 & 0.8 & 1.95 & RY Dra & $3 e-07$ & 10.0 & 10.0 & 1950 & 0.8 & 1.59 \\
\hline T Lyr & 7e-08 & 11.5 & 15.0 & 2050 & 0.8 & 3.26 & NP Pup & $6.5 \mathrm{e}-08$ & 9.5 & 15.0 & 2050 & 0.8 & 1.23 \\
\hline VY Uma & $7 \mathrm{e}-08$ & 6.0 & 15.0 & 2000 & 0.8 & 4.35 & V1942 Sgr & $1.6 \mathrm{e}-07$ & 10.0 & 10.0 & 2000 & 0.8 & 0.23 \\
\hline UX Dra & $1.6 \mathrm{e}-07$ & 4.0 & 10.0 & 1900 & 0.8 & 0.5 & & & & & & & \\
\hline
\end{tabular}


Table C.2. Observational set of O-rich outflows with their observed mass-loss rates, $\dot{M}\left[\mathrm{M}_{\odot} \mathrm{yr}^{-1}\right]$, and expansion velocities, $v_{\infty}\left[\mathrm{km} \mathrm{s}^{-1}\right]$, from Olofsson et al. (2002), González Delgado et al. (2003), and Danilovich et al. (2015) and the assumed drift velocities, $v_{\mathrm{drift}}\left[\mathrm{km} \mathrm{s}^{-1}\right]$. The values of the free parameters of the dust temperature profile $T_{\mathrm{dust}, *}$ and $s$, together with the chi square value of each fit, are also listed.

\begin{tabular}{|c|c|c|c|c|c|c|c|c|c|c|c|c|c|}
\hline Name & $\dot{M}$ & $v_{\infty}$ & $v_{\text {drift }}$ & $T_{\text {dust }, *}$ & $s$ & $\chi^{2}$ & Name & $\dot{M}$ & $v_{\infty}$ & $v_{\text {drift }}$ & $T_{\text {dust }, *}$ & $s$ & $\chi^{2}$ \\
\hline RY CrB & $4 \mathrm{e}-07$ & 5.7 & 10.0 & 950 & 1.3 & 35.99 & $\mathrm{~L}_{2}$ Pup & $1.4 \mathrm{e}-08$ & 2.0 & 15.0 & 950 & 1.3 & 33.19 \\
\hline FZ Hya & $2 \mathrm{e}-07$ & 7.8 & 10.0 & 950 & 1.3 & 34.69 & AZ UMa & $2.5 \mathrm{e}-07$ & 4.5 & 10.0 & 950 & 1.3 & 35.41 \\
\hline IRC+50137 & $1 \mathrm{e}-05$ & 17.0 & 5.0 & 950 & 1.3 & 40.38 & IK Tau & $3 \mathrm{e}-05$ & 18.5 & 5.0 & 950 & 1.3 & 55.88 \\
\hline RX Lac & $8 \mathrm{e}-08$ & 2.2 & 15.0 & 950 & 1.3 & 34.73 & UU Dra & $5 e-07$ & 8.0 & 10.0 & 950 & 1.3 & 35.21 \\
\hline IRC-30398 & $6 \mathrm{e}-06$ & 16.0 & 10.0 & 950 & 1.3 & 39.71 & WX Psc & $1.1 \mathrm{e}-05$ & 19.3 & 5.0 & 950 & 1.3 & 41.92 \\
\hline TZ Aql & $1 \mathrm{e}-07$ & 4.8 & 15.0 & 950 & 1.3 & 33.37 & UX Sgr & $1.5 \mathrm{e}-07$ & 9.5 & 10.0 & 950 & 1.3 & 34.63 \\
\hline AK Hya & $1 \mathrm{e}-07$ & 4.8 & 15.0 & 950 & 1.3 & 33.37 & T Mic & $8 \mathrm{e}-08$ & 4.8 & 15.0 & 950 & 1.3 & 34.6 \\
\hline V1943 Sgr & $9.9 \mathrm{e}-08$ & 6.5 & 15.0 & 950 & 1.3 & 35.07 & FK Hya & $6 \mathrm{e}-08$ & 8.7 & 15.0 & 950 & 1.3 & 33.29 \\
\hline RW Vir & $1.5 \mathrm{e}-07$ & 7.0 & 10.0 & 950 & 1.3 & 33.5 & SW Vir & $4 \mathrm{e}-07$ & 7.5 & 10.0 & 950 & 1.3 & 35.53 \\
\hline BI Car & $3 \mathrm{e}-08$ & 2.2 & 15.0 & 950 & 1.3 & 34.1 & S CMi & $4.9 \mathrm{e}-08$ & 2.0 & 15.0 & 950 & 1.3 & 33.71 \\
\hline R Dor & $1.3 \mathrm{e}-07$ & 6.0 & 10.0 & 950 & 1.3 & 33.6 & TX Cam & $6 e-06$ & 18.5 & 10.0 & 950 & 1.3 & 39.89 \\
\hline TW Peg & $2.5 \mathrm{e}-07$ & 9.5 & 10.0 & 950 & 1.3 & 34.12 & R LMi & $2.6 \mathrm{e}-07$ & 7.5 & 10.0 & 950 & 1.3 & 35.29 \\
\hline S Pav & $8 \mathrm{e}-08$ & 9.0 & 15.0 & 950 & 1.3 & 32.89 & CZ Ser & $8 \mathrm{e}-07$ & 9.5 & 10.0 & 950 & 1.3 & 37.32 \\
\hline CS Dra & $6 \mathrm{e}-07$ & 11.6 & 10.0 & 950 & 1.3 & 34.78 & RX Lep & $5 e-08$ & 3.5 & 15.0 & 950 & 1.3 & 33.98 \\
\hline V744 Cen & $1.3 \mathrm{e}-07$ & 5.3 & 10.0 & 950 & 1.3 & 33.91 & V352 Ori & $5 e-08$ & 8.4 & 15.0 & 950 & 1.3 & 33.74 \\
\hline SY Lyr & $6 \mathrm{e}-07$ & 4.6 & 10.0 & 950 & 1.3 & 38.71 & V PsA & $3 e-07$ & 14.4 & 10.0 & 950 & 1.3 & 33.37 \\
\hline UY Cet & $2.5 \mathrm{e}-07$ & 6.0 & 10.0 & 950 & 1.3 & 35.77 & SV Peg & $3 \mathrm{e}-07$ & 7.5 & 10.0 & 950 & 1.3 & 35.25 \\
\hline RX Boo & $5 e-07$ & 9.3 & 10.0 & 950 & 1.3 & 34.98 & R Hor & $5.9 \mathrm{e}-07$ & 4.0 & 10.0 & 950 & 1.3 & 37.94 \\
\hline CE And & $5 e-07$ & 10.5 & 10.0 & 950 & 1.3 & 36.73 & AB Aqr & $1.3 \mathrm{e}-07$ & 4.2 & 10.0 & 950 & 1.3 & 36.22 \\
\hline V1111 Oph & $1.2 \mathrm{e}-05$ & 17.0 & 5.0 & 950 & 1.3 & 42.79 & RW Lep & $5 e-08$ & 4.4 & 15.0 & 950 & 1.3 & 33.6 \\
\hline W Hya & $8 \mathrm{e}-08$ & 6.5 & 15.0 & 950 & 1.3 & 33.78 & RV Cam & $2.5 \mathrm{e}-07$ & 5.8 & 10.0 & 950 & 1.3 & 35.6 \\
\hline Y Scl & $1.3 \mathrm{e}-07$ & 5.2 & 10.0 & 950 & 1.3 & 34.11 & AFGL 292 & $2.1 \mathrm{e}-07$ & 8.5 & 10.0 & 950 & 1.3 & 34.46 \\
\hline SU Vel & $2 \mathrm{e}-07$ & 5.5 & 10.0 & 950 & 1.3 & 34.73 & SZ Dra & $6 \mathrm{e}-07$ & 9.6 & 10.0 & 950 & 1.3 & 35.21 \\
\hline V Tel & $2 \mathrm{e}-07$ & 6.8 & 10.0 & 950 & 1.3 & 35.76 & R Leo & $1.1 \mathrm{e}-07$ & 8.5 & 10.0 & 950 & 1.3 & 33.71 \\
\hline Y Tel & $5 \mathrm{e}-07$ & 3.5 & 10.0 & 1000 & 1.2 & 39.69 & W Cyg & $1 \mathrm{e}-07$ & 8.3 & 15.0 & 950 & 1.3 & 33.39 \\
\hline BX Cam & $4.4 \mathrm{e}-06$ & 19.0 & 10.0 & 950 & 1.3 & 38.15 & CW Cnc & $5 e-07$ & 8.5 & 10.0 & 950 & 1.3 & 34.17 \\
\hline SU Sgr & $4 \mathrm{e}-07$ & 9.5 & 10.0 & 950 & 1.3 & 35.3 & IRC +10365 & $3 \mathrm{e}-05$ & 16.2 & 5.0 & 950 & 1.3 & 53.92 \\
\hline GX Mon & $8.4 \mathrm{e}-06$ & 19.0 & 10.0 & 950 & 1.3 & 38.66 & EY Hya & $2.5 \mathrm{e}-07$ & 11.0 & 10.0 & 950 & 1.3 & 34.09 \\
\hline AF Cyg & $8 \mathrm{e}-08$ & 3.5 & 15.0 & 950 & 1.3 & 34.54 & T Ari & $4 \mathrm{e}-08$ & 2.4 & 15.0 & 950 & 1.3 & 34.6 \\
\hline T Cep & $9.1 \mathrm{e}-08$ & 5.5 & 15.0 & 950 & 1.3 & 34.89 & BC And & $2 \mathrm{e}-07$ & 4.0 & 10.0 & 950 & 1.3 & 36.32 \\
\hline SV Aqr & $3 \mathrm{e}-07$ & 8.0 & 10.0 & 950 & 1.3 & 34.35 & S CrB & $2.3 \mathrm{e}-07$ & 7.0 & 10.0 & 950 & 1.3 & 35.92 \\
\hline KU And & $9.4 \mathrm{e}-06$ & 20.0 & 10.0 & 950 & 1.3 & 42.04 & UX And & $4 \mathrm{e}-07$ & 12.8 & 10.0 & 950 & 1.3 & 35.75 \\
\hline RS And & $1.5 \mathrm{e}-07$ & 4.4 & 10.0 & 950 & 1.3 & 36.61 & S Dra & $4 \mathrm{e}-07$ & 9.6 & 10.0 & 950 & 1.3 & 35.77 \\
\hline g Her & $1 \mathrm{e}-07$ & 8.4 & 15.0 & 950 & 1.3 & 33.15 & IRC-10529 & $2.5 \mathrm{e}-06$ & 12.0 & 10.0 & 950 & 1.3 & 37.99 \\
\hline Y UMa & $1.5 \mathrm{e}-07$ & 4.8 & 10.0 & 950 & 1.3 & 35.75 & BK Vir & $1.5 \mathrm{e}-07$ & 4.0 & 10.0 & 950 & 1.3 & 34.35 \\
\hline R Hya & $3 \mathrm{e}-07$ & 7.0 & 10.0 & 950 & 1.3 & 35.3 & RR Aql & $2.4 \mathrm{e}-06$ & 9.0 & 10.0 & 950 & 1.3 & 38.29 \\
\hline SS Cep & $6 \mathrm{e}-07$ & 10.0 & 10.0 & 950 & 1.3 & 34.15 & V584 Aql & $5 e-08$ & 2.2 & 15.0 & 950 & 1.3 & 34.09 \\
\hline R Cas & $1.3 \mathrm{e}-06$ & 10.5 & 10.0 & 950 & 1.3 & 37.3 & TU Lyr & $3 e-07$ & 7.4 & 10.0 & 950 & 1.3 & 34.97 \\
\hline EX Ori & $8 \mathrm{e}-08$ & 4.2 & 15.0 & 950 & 1.3 & 34.09 & Theta Aps & $4 \mathrm{e}-08$ & 4.5 & 15.0 & 950 & 1.3 & 32.89 \\
\hline R Crt & $8 \mathrm{e}-07$ & 10.6 & 10.0 & 950 & 1.3 & 35.98 & $\mathrm{IRC}+40004$ & $6 e-06$ & 18.0 & 10.0 & 950 & 1.3 & 42.63 \\
\hline OT Pup & $5 \mathrm{e}-07$ & 9.0 & 10.0 & 950 & 1.3 & 35.41 & RT Vir & $5 e-07$ & 7.8 & 10.0 & 950 & 1.3 & 36.06 \\
\hline AH Dra & $8 \mathrm{e}-08$ & 6.4 & 15.0 & 950 & 1.3 & 33.43 & TY Dra & $6 \mathrm{e}-07$ & 9.0 & 10.0 & 950 & 1.3 & 37.08 \\
\hline$\tau^{4}$ Ser & $1.5 \mathrm{e}-07$ & 14.4 & 10.0 & 950 & 1.3 & 33.72 & U Del & $1.5 \mathrm{e}-07$ & 7.5 & 10.0 & 950 & 1.3 & 33.36 \\
\hline V1300 Aql & $1 \mathrm{e}-05$ & 18.0 & 5.0 & 950 & 1.3 & 41.08 & U Men & $2 \mathrm{e}-07$ & 7.2 & 10.0 & 950 & 1.3 & 36.2 \\
\hline NV Aur & $2.5 \mathrm{e}-05$ & 18.0 & 5.0 & 950 & 1.3 & 52.82 & & & & & & & \\
\hline
\end{tabular}

